\title{
Économie et langue bretonne : un rôle déterminant, deux fois?
}

The economy and the Breton language: a determining role, twice over?

\section{Fañch Broudic}

\section{(2) OpenEdition}

\section{Journals}

Édition électronique

URL : https://journals.openedition.org/lbl/1192

DOI : $10.4000 / \mathrm{lbl} .1192$

ISSN : 2727-9383

Éditeur

Université de Bretagne Occidentale - UBO

\section{Édition imprimée}

Date de publication : 1 juin 2015

Pagination : 153-203

ISBN : 979-10-92331-16-5

ISSN : $1270-2412$

Référence électronique

Fañch Broudic, «Économie et langue bretonne : un rôle déterminant, deux fois ? », La Bretagne Linguistique [En ligne], 19| 2015, mis en ligne le 01 mai 2021, consulté le 22 mai 2021. URL : http:// journals.openedition.org/lbl/1192; DOI : https://doi.org/10.4000/lbl.1192

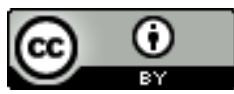

La Bretagne Linguistique est mise à disposition selon les termes de la Licence Creative Commons Attribution 4.0 International. 
Fañch BROUDIC*

\section{Économie et langue bretonne : un rôle déterminant, deux fois ?}

\section{E}

Basse-Bretagne, les villes sont perçues comme des pôles de rayonnement du français dès le $\mathrm{XIX}^{\mathrm{e}}$ siècle, et bien avant. Le breton reste longtemps prédominant comme langue des échanges, en zone rurale notamment. C'est dans la seconde moitié du $\mathrm{XX}^{\mathrm{e}}$ siècle que la révolution économique que connaît alors la Bretagne se traduit en pays bretonnant par une diminution phénoménale du nombre de locuteurs, de l'ordre de $85 \%$ en deux générations. L'explication le plus souvent avancée incrimine l'État central, coupable d'avoir combattu la langue bretonne avec constance et refusé qu'elle soit enseignée. Son rôle a été essentiel, mais a-t-il été suffisant ? Dans un processus de substitution linguistique qui se caractérise par une bien plus grande complexité qu'il n'y parait, le rôle de l'économique a été aussi déterminant, sinon plus, que celui du politique.

Pourrait-il l'être une deuxième fois? En Bretagne, au début du $\mathrm{XXI}^{\mathrm{e}}$ siècle, un nouveau discours valorise la relation entre culture et économie - et c'est également vrai dans d'autres régions, qu'on y

\footnotetext{
* Journaliste, chercheur associé au Centre de recherche bretonne et celtique, UBO. L'auteur remercie Jean Bannier et Malo Bouëssel du Bourg pour les documents qu'ils lui ont transmis.
} 
parle ou non une langue spécifique. La langue bretonne elle-même est désormais présentée comme « une entreprise » de plusieurs centaines de salariés et comme « un marché d'avenir ». Elle s'affiche dans les supermarchés, sur les emballages et dans la publicité. Elle est créditée de modernité, de solidarité, d'authenticité, d'un potentiel positif. Elle suscite, dit-on, la sympathie et l'adhésion de la clientèle. Pour vendre, le breton serait devenu un marqueur identitaire efficace. Ces initiatives lui donnent assurément une nouvelle visibilité. Elles sont parfois présentées implicitement, sinon explicitement, comme pouvant contribuer à un renouveau de la pratique sociale d'une langue que l'on décrit pourtant comme étant sérieusement en danger. En d'autres termes, le monde de l'économie se déclare disponible et volontaire pour la revitalisation de la langue bretonne.

La question qu'induit ce nouveau contexte est de savoir si l'économique peut réellement contribuer à l'inversion du changement de langue. Eu égard aux mutations significatives intervenues au cours des vingt dernières années, la présente communication vise donc à revisiter pour une part l'analyse que j'ai développée sur ce point dans ma thèse sur l'évolution de la pratique du breton de l'Ancien Régime à nos jours, soutenue en $1993^{1}$. J'examinerai ensuite les modalités selon lesquelles tentent de se joindre aujourd'hui le monde de l'économie et celui des promoteurs de la langue : quels sont les postulats mis en avant et les moyens utilisés ? Les publics visés ? Les effets escomptés? Il convient auparavant de procéder à un bref état des lieux de la pratique du breton en rapport avec la connaissance qu'on en a.

\section{Bref historique d'une substitution en trois temps}

Jusqu'au début des années 1990, il était admis qu'on ne pouvait pas, faute de recensements, disposer de données chiffrées sur la pratique du breton ni pour le passé ni pour le présent. Il est vrai qu'avant 1999 il n'était pas d'usage de poser des questions relatives aux pratiques linguistiques dans le cadre des recensements généraux de la population. Les premiers chercheurs qui, à l'image de Jean-

1. Fañch Broudic, La pratique du breton de l'Ancien Régime à nos jours, Rennes, Presses universitaires de Rennes, 1995. 
Baptiste Marcellesi, ont contribué à l'émergence de la sociolinguistique en France étaient confrontés à la pénurie de chiffres qui prévalait alors pour toutes les langues de France. Les universitaires bretons eux-mêmes reconnaissaient en être réduits à des conjectures ou à de simples estimations ${ }^{2}$.

Sans exclure qu'elle puisse donner lieu à réexamen ou ne seraitce qu'à des compléments, la chronologie de la substitution est désormais bien établie. Les récits de voyageurs, les monographies et les enquêtes effectuées ponctuellement ou à l'occasion des polémiques du passé, de multiples ressources archivistiques ou imprimées, les sondages réalisés depuis les années 1970, bien que de nature souvent différente, constituent, dans le cadre d'une démarche de sociolinguistique historique, un matériau suffisamment concret et consistant pour fonder une périodisation en trois temps ${ }^{3}$ :

- Jusqu'aux débuts du $\mathrm{XX}^{\mathrm{e}}$ siècle, l'usage du breton est général. Une part importante de la population rurale se montre longtemps réfractaire ou indifférente à l'égard de la langue nationale. Il n'y a alors que les classes aisées qui aspirent à la connaissance du français. Celui-ci n'est réellement implanté que dans les villes les plus importantes. Jusqu'à la Première Guerre mondiale, le monolinguisme de langue bretonne concerne la moitié de la population de la Basse-Bretagne.

- Le désir de français s'impose ensuite, au point que les bretonnants eux-mêmes en viennent à choisir une autre langue que celle dans laquelle ils s'expriment habituellement, pour élever leurs propres enfants. Les années décisives se situent aux lendemains de la Seconde Guerre mondiale, et c'est à ce moment-là que se résorbe le monolinguisme de langue bretonne. Et c'est dans les années 1960 que le breton cesse d'être connu de la majorité de la population de la BasseBretagne.

- Le breton est désormais une langue minoritaire sur son propre territoire. Si, sur l'ensemble de la Bretagne, le nombre de bretonnants s'élevait à un peu plus de 200000 personnes lors du sondage réalisé en 2007 par l'institut TMO Régions, en

2. Fañch BROUdic, ibid., p. 18-19.

3. Fañch BROUdIC, ibid., p. 347-351 et 441-442. 
Basse-Bretagne il a régressé de $30 \%$ par rapport à la même enquête effectuée dix ans auparavant et le nombre de locuteurs n'est plus à cette date que de 172000 personnes. Le taux de bretonnants a lui aussi diminué sur la même période, passant de $20 \%$ à $13 \%$ s'exprimant très bien ou assez bien en breton.

Il convient d'ajouter qu'au cours de la période qui s'étend des années 1950 à aujourd'hui, la pratique sociale du breton a connu une érosion spectaculaire : en passant de 1100000 locuteurs en Basse-Bretagne à la fin de la dernière guerre à 170000 en 2007, la diminution est de $85 \%$ sur deux générations.

Même s'ils ont mis un certain temps à s'imposer, tous ces constats sont généralement partagés aujourd'hui. Les données cidessus ne sont cependant pas les seules à prendre en compte. L'âge est depuis déjà longtemps le principal caractère discriminant : les bretonnants de plus de 60 ans représentent $70 \%$ d'une population qui ne se renouvelle pas, si ce n'est par l'école, et la pyramide des âges est inversée. Dans la tranche d'âges des 15-19 ans, le taux de locuteurs a grimpé à $4 \%$ par rapport au précédent sondage de 1997, alors qu'il n'est que de $2 \%$ dans celle des 20-39 ans et qu'il s'élève à $46 \%$ dans celle des plus de 75 ans. On observe par ailleurs que la pratique occasionnelle est largement prédominante, seules 35000 personnes (soit $20 \%$ des locuteurs) déclarant parler le breton tous les jours.

En elles-mêmes, ces données soulignent que la substitution n'est cependant pas parvenue à son terme. Même si la transmission intergénérationnelle n'est plus guère assurée et même si le breton n'est plus aujourd'hui un moyen de communication comme le sont le plus souvent les autres langues, son usage correspond toujours à une pratique sociale avérée et la question de la langue bretonne reste incontournable. Alors que le quart des langues du monde a moins de 1000 locuteurs, il figure parmi les 1114 langues sur plus de 6000 recensées à travers le monde qui sont parlées par plus de 100000 locuteurs ${ }^{4}$.

4. Louis-Jean CALVET, Il était une fois 7000 langues, Paris, Fayard, 2011, p. 255. 


\section{La première dimension de l'économique : en faveur de la substitution}

De multiples facteurs ${ }^{5}$ expliquent l'évolution de la pratique sociale du breton en cours depuis le milieu du XIX siècle et qui s'est accélérée dans la seconde moitié du siècle dernier. Ceux qui sont traditionnellement cités ont trait au rôle de l'école depuis l'instauration de l'enseignement primaire obligatoire, à celui des médias depuis l'avènement de la radio et de la télévision, à celui de l'État enfin, accusé d'avoir tout fait pour contribuer à l'éradication de la pratique du breton. Un récent ouvrage de vulgarisation, certes caricatural, assez représentatif néanmoins des idées reçues qui prévalent sur la question, fait ainsi état de "siècles de persécution », d'une politique linguistique « qui ne varie pas d'un pouce » et pointe du doigt une "France engoncée dans son monolinguisme incapacitant $(\text { sic })^{6}$. " Le breton véhicule l'image d'une langue interdite, exclue de l'école, des médias, de l'espace public, n'ayant jamais bénéficié d'une reconnaissance officielle de la part des pouvoirs publics, pas d'un statut positif en tout cas. Ces représentations ne sont assurément pas fausses en tout point, même si elles demandent à être discutées. Elles ont l'inconvénient d'occulter les facteurs économiques et sociaux qui ne sont qu'exceptionnellement évoqués, comme s'ils n'avaient eu aucune incidence sur les usages de langue dans la zone de pratique traditionnelle du breton, soit la Basse-Bretagne.

$\mathrm{Au} \mathrm{XIX}^{\mathrm{e}}$ siècle, ce sont pourtant ceux que mettent en avant un certain nombre d'observateurs, comme s'ils ne se faisaient pas trop d'illusion sur la capacité de l'État ni sur celle de l'école à parvenir rapidement au changement de langue escompté. A.-R. Hamon est en 1841 le secrétaire particulier de l'un des ministres les plus importants de la Monarchie de Juillet, François Guizot. Alors que le train ne parviendra à Quimper qu'une vingtaine d'années plus tard, il plaide

5. Cette première partie reprend pour l'essentiel, tout en les reformulant, les développements figurant sur le même sujet dans ma thèse. Pour les références archivistiques ou documentaires des citations reproduites dans le présent article, prière de se reporter à cette publication, sauf indication particulière. Voir Fañch BROUDIC, La pratique du breton de l'Ancien Régime à nos jours, op. cit.

6. Thierry Jigourel, Langue en Basse-Bretagne. Modernité du breton, Romorantin, Communication presse édition, 2010, p. 19, 25 et 43. 
pour le prolongement d'urgence de la voie ferrée vers la Bretagne : «Un chemin de fer apprendra en 10 ans plus de français aux Bretons que les plus habiles instituteurs primaires. " Lorsque le train entre enfin en gare de Quimper le 7 septembre 1863, un anonyme ironique s'en désole au contraire et, conscient que ce sont bien les enjeux de l'époque, invite les Quimpérois à assister «au convoi funèbre des mœurs, coutumes, langage et traditions de la vieille Armorique, décédée aujourd'hui dans la $1900^{\mathrm{e}}$ année de son âge ».

\section{Nouveaux moyens de communication et migrations}

La même année, le ministre de l'Instruction publique, Victor Duruy, diffuse un questionnaire sur la statistique de l'enseignement primaire incluant plusieurs questions sur les «idiomes et patois en usage » hors de l'école et à l'école. Les réponses qui lui sont adressées depuis la Basse-Bretagne insistent curieusement davantage sur l'impact que pourraient avoir les transformations économiques à venir que sur le rôle de l'école pour le changement de langue. L'inspecteur primaire de Brest se dit ainsi persuadé que «l'idiome breton ne fera définitivement place à la langue française que lorsque celle-ci sera devenue dans notre pays la langue des transactions commerciales, ce qui ne peut manquer d'arriver lorsque les voies ferrées actuellement en cours de construction, auront mis nos populations en contact avec le reste de la France, dont elles sont aujourd'hui pour ainsi dire isolées ».

Son homologue à Quimper est également convaincu que « l'établissement des écoles, le perfectionnement des voies de communication, le contact de plus en plus fréquent avec les populations des villes auxquelles leurs relations avec des étrangers ont peu à peu fait perdre l'habitude de parler constamment breton, ont influé et influent encore d'une manière heureuse sur les campagnes ». L'inspecteur d'Académie du Morbihan partage leur point de vue : « déjà le mouvement des affaires, l'ouverture de voies rapides de communication, ont préparé le terrain : en sortant de leur isolement, les populations bretonnes sentent l'inconvénient d'être souvent obligées de recourir à des intermédiaires pour discuter de leurs intérêts ou conclure leurs transactions ».

Pas plus que l'école, l'industrialisation ou le développement de nouveaux moyens de transport n'ont d'effet radical immédiat sur la 
transformation des usages de langue. L'installation de populations immigrées peut cependant l'accélérer. Les forges de Rohan ayant attiré un grand nombre de travailleurs dans le centre de la Bretagne, Guillaume Le Jean observe en 1874 que « la langue française a fait une percée terrible jusqu'à Caurel et Perret, à plusieurs lieues au-delà de la limite [linguistique]. C'est que là sont les anciennes usines des Salles et tout l'attirail industriel de la maison de Rohan ». Étudiant la situation sociolinguistique de Carhaix au début du $\mathrm{XX}^{\mathrm{e}}$ siècle, Claude Saulnier note que la langue nationale est déjà bien présente dans la ville au sein de la bourgeoisie et qu'elle bénéficie depuis trois siècles d'un point de diffusion privilégié avec l'école privée catholique des Ursulines. De nouveaux arrivants vont contribuer à son extension et à son implantation dans d'autres couches de la société qui n'y avaient pas encore accès : c'est que les employés venus du grand ouest, de l'Allier ou de la Nièvre travailler à la gare parlent le français. C. Saunier le note dans une formule lapidaire : « la langue du $\mathrm{XX}^{\mathrm{e}}$ siècle, issue de l'industrialisation, est entrée dans Carhaix ». L'acculturation au français peut cependant se faire plus paisiblement. Dans ses mémoires, l'écrivain de langue bretonne Yeun ar Gow [Yves Le Goff], né en 1897, consacre tout un chapitre aux travaux de construction de la ligne de chemin de fer entre Carhaix et Châteaulin. Les responsables du chantier, eux aussi venus de l'extérieur, avaient trouvé à se loger à Pleyben : le jeune Yeun ar Gow en vient à " marmonner quelques mots de français ${ }^{7}$ » tout simplement en jouant avec leurs enfants.

\section{Urbanisation et développement du tourisme}

La croissance des villes reste lente en Basse-Bretagne jusqu'au milieu du $\mathrm{XX}^{\mathrm{e}}$ siècle. Mais le développement urbain a une incidence sur l'évolution sociolinguistique de la région. Bien qu'attirant vers elles des populations monolingues bretonnantes touchées par l'exode rural, Brest, la ville la plus importante du Finistère, et Quimper, le chef-lieu du département, sont plus francophones que les autres villes : les comparants en justice qui en sont issus n'ont

7. « [D] a zrailha tammou galleg. » Yeun AR Gow, E skeud tour bras Sant Jermen, La Baule, Ar Skrev, 1955, p. 62. 
guère besoin de faire appel à l'interprète, alors que ceux des villes de taille inférieure, puisqu'ils ne parlent que le breton, le font bien davantage. Dans les paroisses urbaines, en 1902 comme en 1944, la prédication de langue française s'adresse à des populations plus nombreuses que celle en breton. Tout au long du XIX ${ }^{\mathrm{e}}$ siècle et dans la première moitié du $\mathrm{XX}^{\mathrm{e}}$, la langue nationale a d'abord conquis les villes, avant qu'elles ne deviennent elles-mêmes des centres de propagation du français. Puis, elle a envahi les bourgs, vers le milieu du XX $\mathrm{X}^{\mathrm{e}}$ siècle, pour se diffuser ensuite vers les campagnes. À chaque étape, le rayonnement s'est fait par cercles concentriques du centre vers la périphérie.

La diffusion d'une langue ne tient cependant pas qu'au rayonnement urbain ni à la géographie: pour réussir, elle doit aussi bénéficier du concours de certaines catégories sociales qui jouent le rôle d'intermédiaires linguistiques. L'observation d'un enquêteur en 1946 est tout à fait symptomatique à cet égard : à Plouescat, dans le commerce, écrit-il, « il n'est plus nécessaire de connaître le breton, même pour le commerce avec les gens de la campagne, mais il est bon et prudent de connaître cette langue ${ }^{8} \gg$. D'après P. Keraval, c'est au contact des touristes que les commerçants du pays bigouden en viennent les premiers à la pratique du français autour des années 1950 : le reste de la population les suivra.

Au même moment, les paysans emploient toujours de préférence le breton dans les communes rurales du Léon et ailleurs : il reste leur langue de travail, ainsi que celle de leurs échanges avec les commerçants et les artisans, qu'ils soient coiffeur, médecin ou notaire, tout comme à la mairie. Mais l'usage du français tend à s'imposer dans certaines administrations comme la perception, la gendarmerie ou la poste, dont de nombreux agents connaissent pourtant la langue régionale. Les paysans « ne parlent français que lorsqu'ils sont contraints par une affaire à traiter » ou lorsqu'ils rencontrent « un étranger» ne faisant pas partie de leurs relations habituelles. En 1946, les commerçants et les artisans, considérés comme « une population d'un rang social plus élevé » ont déjà adopté le français dans le cadre de leur vie familiale et personnelle, mais ils savent s'adapter et optent pour l'une ou l'autre langue en fonction de leur clientèle. Et c'est ici

8. Les termes soulignés l'ont été par l'enquêteur. 
qu'interviennent les variables âge et sexe : à Guissény, par exemple, les jeunes commerçants et leurs enfants, surtout les filles, " feignent d'ignorer le breton et parlent en français même aux paysans ». Cette donnée va s'accentuer avec le temps.

Dans les années d'après-guerre, la population rurale est en diminution, la perte de population est particulièrement importante dans l'intérieur. La Bretagne se cherche un nouvel avenir et les mouvements de population vont y prendre une nouvelle importance. Elle est de toutes les régions françaises celle qui connaît la plus forte émigration en chiffres absolus. Mais tous les migrants ne quittent pas leur région. Au début des années 1950, le quart de la population bretonne change certes de commune de résidence, mais si un tiers des migrants s'installe dans d'autres régions, les deux-tiers quittent leur commune de résidence pour rejoindre une plus ou moins grande ville à l'intérieur même de la région. La Bretagne reste alors une région de forte natalité et de familles nombreuses : la perspective d'une émigration ou d'une migration considérées comme inéluctables est perçue comme une incitation à l'apprentissage de la langue nationale. Le breton est considéré comme un handicap. L'accès aux emplois de l'émigration ou de la ville, que ce soit ceux de la fonction publique ou ceux de l'industrie, implique la meilleure connaissance possible du français et les jeunes parents l'adoptent sans hésiter pour s'adresser à leurs enfants. L'exode linguistique et l'exode rural sont deux réalisations parallèles d'une même situation de crise.

La Bretagne connaît simultanément une immigration d'un nouveau type, liée au tourisme. Ce dernier a été au départ l'apanage de voyageurs isolés, tel Mérimée, se faisant éventuellement accompagner d'un interprète. Il devient un phénomène de masse dès la fin du $\mathrm{XIX}^{\mathrm{e}}$ siècle, non seulement dans les nouvelles stations balnéaires qui surgissent le long de la côte, mais aussi dans l'intérieur : selon Yeun ar Gow, « lorsque des étrangers venaient chercher une voiture, il leur fallait à chaque fois un chauffeur sachant le français ${ }^{9} \gg$. Comme rares sont alors les cochers pouvant s'exprimer correctement en français,

9. «[P] zene divroidi da glask eur wetur, e falveze ganto bewech kaout eur bleiner hag a ouie galleg. " Il faut comprendre une voiture à cheval, à louer chez son père, à Pleyben. Yeun AR Gow, op. cit., p. 49. 
il n'est pas facile de répondre à la demande ${ }^{10}$. Mais puisque ce sont généralement les vacanciers qui imposent l'usage de leur langue partout où ils passent, le clergé lui-même apprend à s'adapter : dès 1902, il assure la prédication en français en de nombreuses paroisses pendant « la saison des bains ». Le phénomène s'accentue lors des congés payés de 1936, puis dans les années 1950, mettant un nombre toujours croissant de visiteurs en contact direct avec l'habitant, quel que soit son statut (commerçant, hôtelier, loueur, salarié...). Michel Le Bris présente les touristes comme « les voyageurs de commerce de la modernité ».

\section{Les guerres : des temps de perturbation linguistique}

La conscription et les guerres donnent également lieu à de multiples déplacements, non seulement de militaires, mais également de civils. Le service militaire devient obligatoire en 1872. Pendant leur service, les jeunes bretonnants de sexe masculin souffrent souvent de leur méconnaissance du français. La conscription impliquant l'éloignement du milieu d'origine, elle a nécessairement contribué à la diffusion de la langue nationale. En 1863, l'inspecteur primaire de Brest considère ainsi que «la loi sur le recrutement de l'armée appelle chaque année sous les drapeaux un certain nombre de jeunes gens qui reviennent dans leur commune avec une certaine connaissance du français ». Mais une fois de retour chez eux, le breton redevenait leur moyen usuel d'expression.

La guerre 14-18 est considérée comme un moment clé dans l'évolution sociolinguistique de la Basse-Bretagne. La raison généralement invoquée en est le séjour prolongé de dizaines de milliers de jeunes Bretons bretonnants sur le front, qui induit par voie de conséquence un contact durable avec la langue française. Curieusement, les témoignages dont on dispose insistent davantage sur leur pratique usuelle du breton au front. L'écrivain Marc Bloch rapporte par exemple que « le recrutement les avait pris aux quatre coins de la Bretagne, si bien que chacun parlait un dialecte différent, ceux d'entre eux qui savaient un peu de français ne pouvaient que rarement servir d'interprètes auprès des autres ». Alain Roignant, un agriculteur originaire de Sizun affecté sur le front d'Italie, compose en breton

10. Yeun Ar Gow, ibid. 
des chansons nostalgiques sur les jeunes filles de son pays ${ }^{11}$. Le journal L'Écho du boqueteau raconte en juin 1918 que des Provençaux viennent d'être versés dans un régiment de Bretons : « [ils] parlent une langue à laquelle nous n'entendons rien. Est-ce à nous de les en blâmer ? " Il est incontestable que les Bas-Bretons ont continué à s'exprimer en leur langue sur le front.

Ils le font même parfois à l'écrit. L'abbé Conseil, originaire de Cléder, rédige quelques-unes de ses lettres en breton. Les cartes postales qu'adresse René Noël Abjean à sa famille, à Plouguerneau, sont parfaitement rédigées en français, bien qu'entrecoupées de phrases en breton, et il lui arrive pour cela d'être censuré. Un bureau spécial du service de contrôle postal aux armées supervise effectivement les correspondances en breton. Il n'en reste pas moins que les lettres qu'écrivent ces soldats à leur famille sont généralement rédigées en français. Ce qui s'explique bien par la chronologie : les plus jeunes des soldats et des marins mobilisés en août 1914 ont été alphabétisés en français, puisqu'ils sont nés en 1894 et qu'ils ont fréquenté l'école, obligatoire depuis une douzaine d'années déjà. D'après Pierre-Jakez Hélias, ils occupaient leurs jours de repos à l'arrière « à dévorer tous les imprimés français qui leur tombaient sous la main ${ }^{12} »$. La Bretagne, bien qu'éloignée du front, vit tout de même pendant quatre ans au rythme de la guerre. À l'arrière aussi, les incidences du conflit sur les usages de langues sont mesurables : en 1917, le curé doyen de la paroisse Saint-Louis de Lorient déclare qu'il lui est « impossible de prêcher en breton » depuis son déclenchement, et le recteur de Keryado qu'il doit prêcher en français «à toutes les messes » depuis le même moment.

Le scénario se reproduit au cours de la Seconde Guerre mondiale, en s'étendant géographiquement. À Langonnet, le recteur entreprend de prêcher en français à partir de l'exode des Lorientais. Des « messes des réfugiés » sont dites dans de nombreuses paroisses du Vannetais (Melrand, Locmalo, Saint-Tugdual...), tout comme dans l'évêché voisin de Quimper et Léon : il y a désormais à

11. Alain RoignAnT, « Mouez eur paotr pell euz e vro », Brud nevez, n²71, 2008, p. 48-51.

12. Pierre-Jakez HÉLiAs, Le cheval d'orgueil. Mémoires d'un breton du pays bigouden, Plon, 1975, p. 215. 
Plabennec «des causeries françaises » à destination des réfugiés, mais dont profite aussi la population locale, celle des bourgs en particulier. À Plougastel-Daoulas, la guerre amène des familles nonpaysannes et non-bretonnantes à s'installer pour la première fois à la campagne. Entre 1940 et 1944, les Douarnenistes qui vont se ravitailler à Guengat, à $13 \mathrm{~km}$ de distance, savent peut-être le breton comme ceux chez qui ils se présentent, mais les uns et les autres ont désormais le même français à leur disposition. La langue nationale est confirmée dans sa fonction de moyen de communication extralocal, alors que le breton est perçu comme limitatif, et du même coup relégué à un statut de second rang. En mettant en contact des populations qui l'étaient peut-être moins en temps normal, la guerre aiguise la perception qu'elles peuvent avoir de leur propre pratique linguistique.

\section{Le développement des échanges}

Les échanges de proximité ont toujours existé entre la ville et la campagne environnante. En 1946, un enquêteur observe ainsi que la population de Milizac la plus proche de Brest, à une douzaine de kilomètres de distance, est la moins bretonnante de la commune, d'après lui, « la raison en est bien simple : c'est que ces gens-là ravitaillent la ville en lait, légumes, etc., à raison de deux, trois fois par semaine ${ }^{13}$ », et le vendeur opte pour le français si sa clientèle ne connaît que cette langue. À Guipavas, dont une partie des habitants travaille à l'arsenal de Brest, « le français fait de grands progrès ${ }^{14} »$.

L'évolution technologique joue en même temps en faveur de la langue nationale. La technologie traditionnelle contribue pendant un certain temps à prolonger l'usage du breton : les métiers tels que maréchaux-ferrants, charrons, bourreliers..., en relation directe avec la paysannerie continuent de le parler partout. L'irruption de nouvelles techniques, qu'elles soient en rapport avec les matériels ou économiques, n'implique pas le passage immédiat au français «à cause des termes techniques dont on ne connaît pas l'équivalent » en cette langue, mais ils finiront par s'imposer ${ }^{15}$. Au Juch par exemple,

13. Fañch Broudic, op. cit., p. 149.

14. Fañch BROUDIC, ibid., p.160.

15. Fañch BRoudic, ibid., p. 160. 
le mécanicien s'exprime plutôt en français, de même que le président local du syndicat des agriculteurs. Et si le français prime à Briec-del'Odet, c'est pour une simple... raison de vocabulaire : « les termes techniques ne sont connus qu'en français. Quand on parle des nouveaux systèmes de culture, tout se traite en français, pour la bonne raison que là-dessus il n'y a rien d'écrit en breton, ou du moins qui soit connu ${ }^{16} \gg$. Et c'est en français que les couches les plus modernistes de la JAC (Jeunesse agricole catholique) débattent de l'avenir de l'agriculture régionale, quand bien même l'usage du breton reste alors très important au sein de ce mouvement de jeunesse.

Les mutations observables au niveau microéconomique le sont aussi au niveau macroéconomique. Dans la seconde moitié du $\mathrm{XX}^{\mathrm{e}}$ siècle, le complexe agroalimentaire breton ne cesse de prendre de l'importance, et l'économie bretonne vit intensément une intégration toujours plus poussée au sein de l'économie nationale, européenne et même mondiale. En un demi-siècle, la Bretagne expérimente la plus formidable transformation de son histoire, une sorte de bond en avant à la chinoise que tous les analystes s'accordent à considérer comme une révolution. La Bretagne de l'an 2000 n'a plus grandchose à voir avec celle de 1950. Comme l'écrivent Michel Nicolas et Jean Pihan, « la société bretonne s'est restructurée sur un nouveau mode, se rapprochant globalement de la moyenne française ${ }^{17} \gg$. Et c'est précisément au moment où l'économie régionale vit une telle phase de mutations radicales que la connaissance et l'usage du breton connaissent une régression colossale en perdant quelque 900000 locuteurs en une soixantaine d'années.

La concomitance de l'une et de l'autre transformation n'est pas fortuite : c'est in fine le développement des échanges qui explique l'évolution sociolinguistique extrêmement rapide qu'a vécue la Basse-Bretagne dans la seconde moitié du $\mathrm{XX}^{\mathrm{e}}$ siècle. Ces échanges sont globalement de nature économique, et il convient d'y associer la modernisation des moyens de transport, les mouvements de population, les avancées technologiques, etc. Il faut aussi prendre en compte les échanges intellectuels, avec l'extension de la formation initiale et

16. Fañch BROUdic, op. cit., p. 160.

17. Michel Nicolas et Jean PiHan, Les Bretons et la politique. 30 ans de scrutins en Bretagne, 1958-1988, Rennes, Atelier universitaire de recherche, 1988, p. 253. 
continue, le développement des médias de masse et de la communication notamment. Jean-Yves Guiomar, après avoir noté que « la diffusion de la langue française va de pair avec le développement des rapports marchands », résume son propos en une formule saisissante : « les mots de la langue française collent à la marchandise ${ }^{18}$ ».

Mais plus que d'une concomitance entre les mutations en cours, il serait plus juste de parler d'une forme d'anticipation de la part des locuteurs eux-mêmes. Car c'est dans les années de l'immédiat après-guerre que les jeunes parents font le choix d'élever leurs enfants en français, et non plus en breton comme ils l'avaient été eux-mêmes. De multiples autres signes laissent supposer que toute une organisation sociale se prépare à imploser simultanément : l'arrêt de la lecture collective de Buhez ar Zent [La vie des saints] en breton le soir en famille, l'essor des bals avec ou sans piano mécanique, une demande de plus en plus pressante en faveur de la prédication en français, les attentes des jeunes filles qui jugent le français "plus chic » et "distingué » alors que le breton leur paraît «arriéré ». La langue bretonne, qui était la marque et le moyen d'expression de l'ancienne société, est perçue négativement et ne pouvait pas assurer la transition vers les nouvelles formes d'organisation sociale en gestation. Alors qu'on n'a compris que bien plus tard les effets des comportements adoptés à ce moment-là sur les usages de langues, l'abandon progressif du breton comme moyen de communication habituel a été, non la conséquence, mais l'une des conditions nécessaires de la modernisation économique de la Bretagne. La « révolution silencieuse », en Basse-Bretagne, c'est aussi la substitution, le changement de langue.

\section{La langue bretonne dans l'entreprise}

À cet égard, les représentations liées à la langue bretonne dans le monde de l'économie à la fin du $\mathrm{XX}^{\mathrm{e}}$ siècle sont très clairement exprimées dans une enquête réalisée en mai 1990 par des lycéens guingampais auprès de 200 entreprises du Finistère, des Côtes d'Armor et du Morbihan ${ }^{19}$. Seules $27 \%$ de celles qui ont répondu

18. Émile MASSON, Les Bretons et le socialisme, présenté par Jean-Yves Guiomar, Paris, Maspéro, 1972, p. 33.

19. L'enquête a recueilli un taux de réponse de $50 \%$. Les entreprises concernées 
déclarent l'utiliser au sein de l'entreprise, quand $68 \%$ ne le font pas. Celles qui le font reconnaissent le faire essentiellement dans le domaine commercial, beaucoup moins au niveau du fonctionnement interne ou de l'information produit, et vraiment très peu au niveau de la publicité ou de la correspondance. Si elles le font, c'est dans le but d'améliorer leur image ou pour leur satisfaction personnelle, plus que pour déclencher concrètement une transaction ou l'acte d'achat. Dans une proportion tout à fait minime $(2 \%)$, l'utilisation du breton vise une clientèle bretonnante socialement circonscrite: "souci de service auprès des anciens et besoin[s] d'une clientèle rurale et agricole $»$.

$73 \%$ des entreprises admettent qu'elles ne sont pas du tout intéressées par le breton, pour deux types de raisons :

- Il représente tout d'abord un moyen de communication « restreint»: les produits sont peu adaptés ; les clients et les fournisseurs ne connaissent pas la langue ; le personnel a souvent des difficultés de compréhension; le breton semble réservé au patrimoine culturel et non économique.

- La langue est ensuite perçue comme étant désuète en raison de son peu d'utilité, de son utilisation limitée et de son avenir incertain. L'anglais et l'allemand semblent bien plus indispensables, dans la mesure où la clientèle est désormais touristique et étrangère.

De nombreux facteurs relevant du domaine économique et social ont contribué depuis le $\mathrm{XIX}^{\mathrm{e}}$ siècle à la transformation du paysage linguistique en Basse-Bretagne : les déplacements de population et les migrations saisonnières ou définitives, l'urbanisation, le développement des relations commerciales de proximité, l'intensification des échanges au niveau national comme à l'international, l'évolution technologique aussi. Mais dans la seconde moitié du XX $\mathrm{XX}^{\mathrm{e}}$ siècle, ceux qui se préoccupent du devenir de la langue bretonne concentrent leurs efforts sur le développement de l'enseignement bilingue et de médias en breton. Quand on l'utilise sur le lieu de travail, c'est plus par habitude ou par routine. Et pourtant, des initiatives surprenantes

relevaient du secteur agricole, de ceux du bâtiment ou du secteur secondaire, et pour la moitié d'entre elles du tertiaire. 
font entrer le breton dans la modernité : le 17 novembre 1965, Émile Gustave Morzadec, résidant en Loire-Atlantique, dépose auprès de l'INPI (Institut national de la propriété industrielle) un brevet pour un rasoir à cheveux, réglable à volonté, du nom de " Troher bleo ${ }^{20}$ " (document 1). La donne aurait-elle donc changé ?

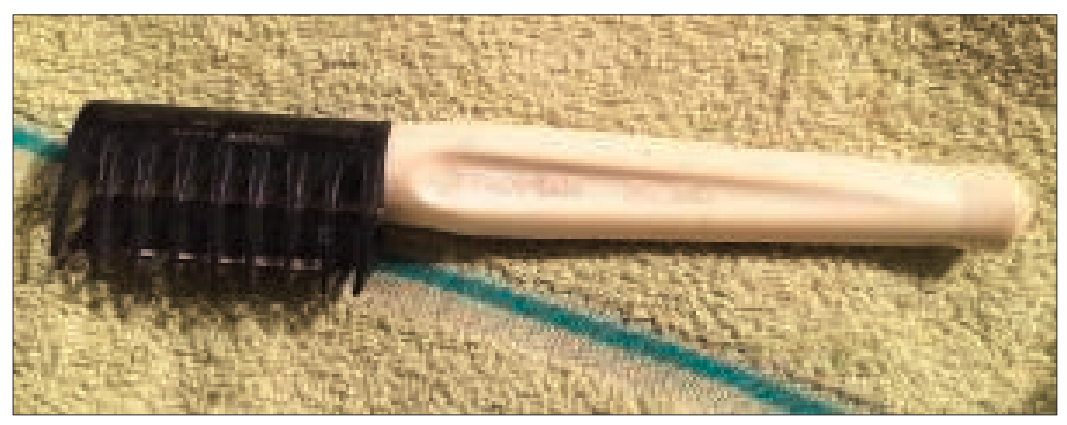

Document 1. Troher bleo. Rasoir à cheveux, dont Émile Gustave Morzadec, résidant en LoireAtlantique, obtient le brevet de l'INPI le 21 novembre 1966. «Troher bleo » : littéralement « coupeur de cheveux», également dénomination du coiffeur en breton. On remarquera que le terme utilise l'orthographe universitaire de la langue bretonne, adoptée une dizaine d'années auparavant.

\section{La nouvelle dimension de l'économique : vers l'inversion du changement de langue?}

$\mathrm{Au}$ début du $\mathrm{XXI}^{\mathrm{e}}$ siècle, l'impression prévaut que la langue bretonne est désormais partout présente : sur les panneaux de signalisation routière, dans les écoles, mais aussi dans le commerce, dans les moyens de transport, dans la presse. " Moins on le parle, plus on en parle ", écrivait déjà le journaliste Didier Eugène dans le journal Ouest-France le $1^{\mathrm{er}}$ août 1991. Le propos serait-il toujours pertinent? Moins on le parle, plus il s'affiche, pourrait-on dire plus justement aujourd'hui, à en juger par la manière dont il est présent dans des grandes surfaces, sur des produits alimentaires, voire dans la publicité. Ces initiatives contribuent effectivement à lui assurer

20. Ce «Troher bleo » [coupe-cheveux] vient de faire l'objet d'un don au Musée de Bretagne à Rennes, de la part de Mme Jezegou, de Saint-Servais. Remerciements à Laurence Prod'homme, conservatrice au Musée, pour les informations qu'elle a collectées sur cet objet. 
une visibilité qu'il n'avait pas antérieurement. Elles se présentent généreusement comme visant à la promotion de la langue bretonne. Elles s'insèrent aussi dans un discours plus général qui valorise désormais la relation entre culture et économie.

« L'investissement dans la culture est non seulement nécessaire socialement, mais aussi indispensable économiquement [...]. Je ne crois pas qu'il faille opposer culture et économie. Tout investissement dans la culture est aussi l'occasion de retombées économiques ${ }^{21}$. " Ces déclarations à la presse d'Hervé Digne, un des fondateurs du Forum d'Avignon ${ }^{22}$, s'inscrivent dans un mouvement général et sont symptomatiques de réflexions qui ont pris une importance croissante dans le débat public au cours de la dernière période. En décembre 2013, un rapport de l'Inspection générale des finances traitait précisément de l'apport de la culture à l'économie en France : il l'évalue à $104,5 \mathrm{Md} €$, estimant qu'elle contribue pour 3,2\% à la richesse nationale et qu'elle emploie 670000 personnes. Selon le rapport, il existe une corrélation positive entre les initiatives culturelles et le développement local ${ }^{23}$. Différentes recherches ont par ailleurs entrepris d'explorer le lien entre cultures régionales et développement économique ${ }^{24}$, il n'est donc pas surprenant qu'en Bretagne aussi ces problématiques soient devenues prégnantes ${ }^{25}$.

Mais est-il forcément question de langue lorsqu'on évoque la culture ? Dans le cadre national, la question ne se pose guère. Dès que le débat porte sur la culture dite « régionale », par contre, la

21. Propos recueillis par Harry BeLlet, Le Monde, 8 août 2014, p. 10.

22. Le Forum d'Avignon se définit comme un laboratoire d'idées au service de la culture, dont l'objectif est d'approfondir les liens entre les mondes de la culture et de l'économie au niveau international, européen et local. Il vise à explorer tant la dimension économique de la culture que le rôle de cohésion sociale et de création d'emplois des secteurs culturels. Il réunit plus de 400 acteurs : artistes, dirigeants d'entreprise, écrivains, réalisateurs, universitaires... http://www. forum-avignon.org/fr/le-forum-davignon. Consulté le 22 janvier 2014.

23. Serge KANCEL \& alii, L'apport de la culture à l'économie en France, décembre 2013. Téléchargeable sur https://www.economie.gouv.fr/files/03-rapport-igfigac-culture-economie.pdf.

24. Voir notamment Jean-Robert Alcaras, Philippe BLANChET et Jérôme JouberT (dir.), Cultures régionales et développement économique, Actes du colloque d'Avignon, 5 au 6 mai 2000, Annales de la Faculté de droit d'Avignon, 2001.

25. Le Conseil culturel de Bretagne a lancé en 2013 une enquête sur le lien entre culture et économie dans la région. 
dimension linguistique interfère aussitôt, ce qui n'est guère surprenant dans la mesure où dans le langage courant comme dans celui des décideurs « la langue fait partie intégrante de la culture » et qu'elle est reconnue dans le cas du breton comme « un des fondamentaux de la culture bretonne ${ }^{26} \gg$. Autrement dit, le breton n'est plus vraiment perçu comme un moyen de communication, c'est la langue en ellemême qui devient un élément de la culture régionale et se trouve dès lors patrimonialisée dans la représentation qu'on en a. Alors que la langue est ordinairement le moyen par lequel s'exprime une culture, dans le cas du breton l'idée que l'on s'en fait est désormais inversée ${ }^{27}$. Des interférences existent entre langue et culture, qu'elles soient « régionales » ou non, mais les deux termes ne peuvent être considérés comme synonymes. René Kahn fait cependant remarquer que le syntagme « culture régionale » est un désignatif polysémique et que la langue peut en être un trait constitutif et y jouer « un rôle singulier ${ }^{28} »$.

Document 2. Le numéro hors-série de la revue ArMen, paru en février 2012. À la une, une photo de Steven Guéguéniat, PDG de l'imprimerie Ouestélio, à Brest. On remarquera que graphiquement le sous-titre «brezhoneg \& business » est en grisé sur fond de couleur, mais en plus gros caractères, et de ce fait moins agressif que le surtitre "Langue bretonne et entreprise», en lettres rouges sur fond blanc. II n'en paraît pas moins comme une forme de slogan programmatique pour deux raisons au moins : d'une part, il n'inclut aucun terme de français (mais pas au point de l'exclure totalement puisqu'il est fortement présent par ailleurs sur la page de couverture), d'autre part, il affiche une association peu courante entre un terme de breton qui n'est pas inconnu (et qui dans le contexte de la page peut se décoder aisément) et l'anglais archiconnu « business ».

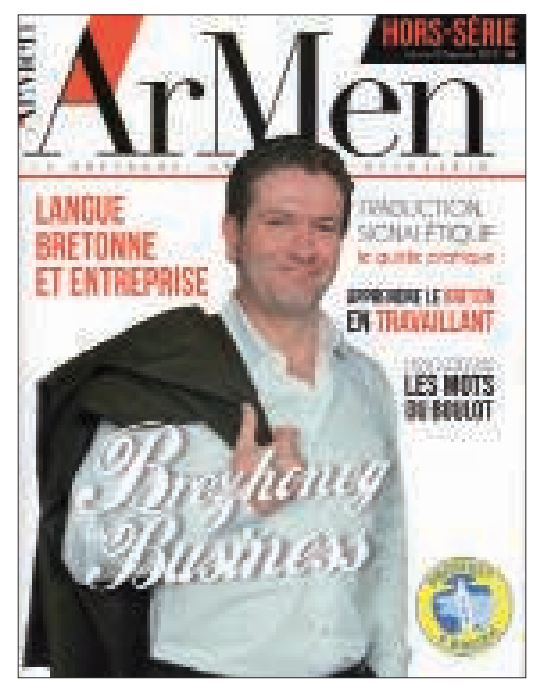

26. Langue bretonne et entreprise. Brezhoneg \& business, ArMen, hors-série, février 2012, p. 35.

27. Fañch BROUDIC, Parler breton au XXIe siècle. Le nouveau sondage de TMORégions, Brest, Emgleo Breiz, 2009, p. 156.

28. René KAHN, «Territoires et modalités du dialogue économie - culture », Langues régionales, cultures et développement, Dominique Huck et René Kahn (dir.), Paris, L'Harmattan, 2009, p. 31-32. 
La chronologie permet de suivre l'émergence de cette thématique telle que l'a promue un numéro hors-série de la revue ArMen en 2012 sous le titre accrocheur «Brezhoneg \& business » (document 2). D'après Yann Rivalain ${ }^{29}$, réconcilier l'économie et la culture serait un « rêve des années 1980 », quand un hypermarché de Lanester expérimente pour la première fois la mise en place d'une signalisation bilingue, qui est d'ailleurs assez vite retirée. Une dizaine d'années plus tard, l'Intermarché de Plouguerneau renouvelle plus durablement l'expérience et suscite des émules (documents 3 et 4).

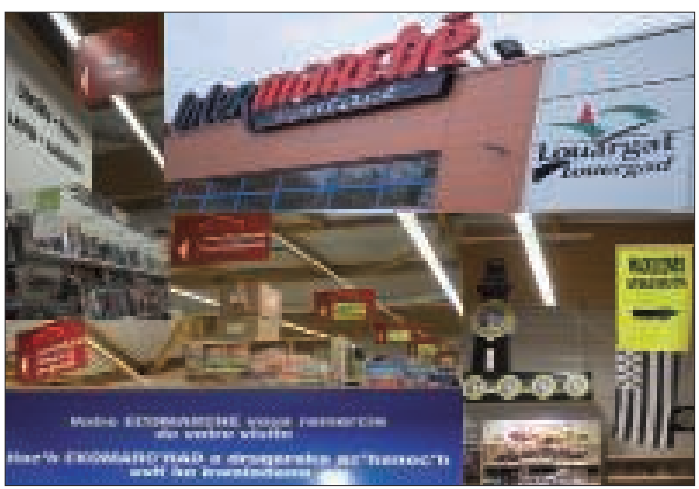

Document 3.

L'enseigne Intermarché de Louargat, aujourd'hui. Le supermarché est en attente de transformation de ses rayonnages, ce qui explique l'abondance des indications sur fond jaune, qui sont toutes rédigées en français.

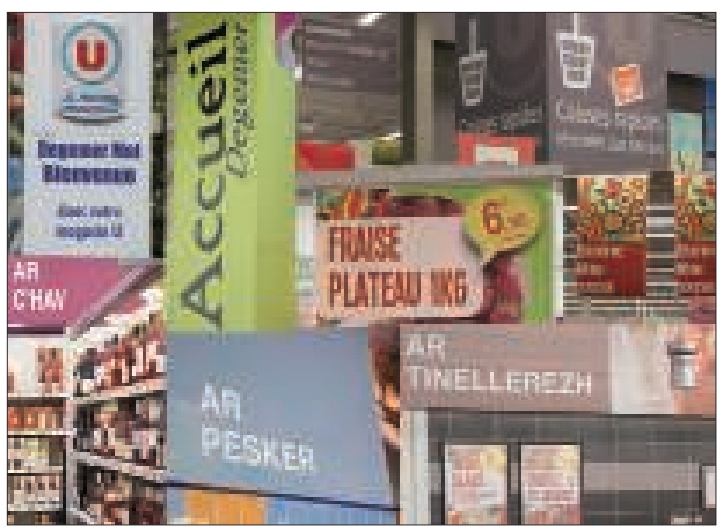

Les grands « secteurs » du supermarché ne sont renseignés que par des libellés en breton, exclusivement. Les rayons sont indiqués par un terme breton, placé en gras au-dessus de ceux en français, plus nombreux et donc plus explicites. Les promotions, les événements et les caisses sont rédigés uniquement en français. Le bilinguisme intégral serait-il envisageable?

Document 4. L'enseigne Super U du quartier de Keredern, à Brest.

29. Yann Rivallain, « Produit en breton / Produet e Breizh », ArMen, op. cit. 
Entre-temps, le premier organisme de formation longue à la langue bretonne s'est créé en 1986 sous l'appellation de Stumdi : son objet principal, que partagent aujourd'hui plusieurs autres organismes, est d'enseigner le breton dans le cadre de formations intensives de six à neuf mois à destination des adultes. À la même période, diverses associations et quelques individualités motivées mettent sur pied de véritables PME (petites et moyennes entreprises) culturelles dans les domaines de l'édition, de la musique, du spectacle vivant ou des médias. Si elles peuvent le faire, c'est aussi parce que le Conseil régional a progressivement renforcé sa politique de soutien aux langues et cultures de Bretagne, dans un premier temps via l'Institut culturel de Bretagne. Puis, l'implication de la région va se concrétiser le 14 décembre 2004 par l'adoption d'un premier plan de politique linguistique reconnaissant officiellement le breton et le gallo comme langues de Bretagne.

\section{L'émergence de "Produit en Bretagne"}

Analysant les groupements localisés d'entreprise, Sandrine Stervinou et Sébastien Lê présentent comme « une gageure » la création de « Produit en Bretagne » en 1993 par quatre cadres d'importantes entreprises finistériennes ${ }^{30}$. Le concept d'une marque régionale commune est innovant et son développement va devenir presque une saga. Le projet séduit en effet tant et si bien que deux ans plus tard l'association " Produit en Bretagne » (par la suite : PeB) regroupe 70 adhérents, puis 171 en $2006^{31}$ et jusqu'à 300 en $2014^{32}$ : ces chiffres représentent approximativement un doublement tous les dix ans. Si l'on en croit son site internet tout comme ce qui généralement s'écrit à son sujet, PeB est une association de chefs d'entreprise « qui

30. Les membres fondateurs sont Jean-Claude Simon (groupe Even), Claude Pujol (magasins Leclerc), Jean Bannier (Crédit mutuel de Bretagne) et JeanYves Chalm (Le Télégramme), voir Sandrine Stervinou et Sébastien LÊ, «Une méthodologie pour analyser les groupements localisés d'entreprises le cas de l'association 'Produit en Bretagne'», Langues régionales, cultures et développement, op. cit., p. 171-173.

31. S. STERVINOU et S. LÊ, ibid., p. 172.

32. D'après le site http://www.produitenbretagne.bzh/ consulté le 2 octobre 2014. 
ont à cœur de valoriser leur région ${ }^{33} »$. Les 300 entreprises adhérentes déclarent employer un total de 100000 salariés.

Le but clairement affiché est d'inciter à l'achat de produits fabriqués en Bretagne, de manière à favoriser l'emploi régional et le développement des entreprises concernées. Le fameux logo jaune avec un phare en surimpression sur fond de carte de Bretagne stylisée assure désormais la promotion de plusieurs milliers de produits fabriqués dans la région. Ce logo affiche un taux de notoriété proche de $100 \%$ en Bretagne et de $50 \%$ en Île-de-France. PeB mène périodiquement des campagnes publicitaires ou d'information pour inciter le consommateur à "relocaliser» ses achats : du 14 juillet au 15 août 2014, elle a ainsi organisé une tournée dans 28 stations littorales de Bretagne sud dans le but de promouvoir «l'achat de proximité ${ }^{34} \gg$. Sandrine Stervinou et Sébastien Lê listent les bénéfices qu'escomptent les adhérents de leur adhésion à PeB : accroissement des ventes, fidélisation du consommateur, amélioration de l'image de l'entreprise en interne et en externe, référencement dans la grande distribution, etc. Comme l'association est généralement présentée comme « la » référence en matière de produits bretons, l'aventure apparaît dans les médias comme une success story à la bretonne, au point que d'autres régions françaises ou européennes, comme la Catalogne, songent à en reprendre le modèle ${ }^{35}$.

Pourtant, selon les deux chercheurs, tout n'a pas toujours été simple au cours des 20 ans d'existence de la structure. Elle a tout d'abord été confrontée à un double problème : la sortie plutôt discrète de certains adhérents qui décident de la quitter ${ }^{36}$ et surtout l'augmentation continue du nombre de membres, des entreprises ne concevant pas de ne pas y être dans la mesure où leurs confrères et voisins y étaient. Une telle croissance a pour effet de générer " une concurrence intrinsèque » au sein du réseau lui-même à propos de produits de même nature ${ }^{37}$. Par ailleurs, écrivent-ils, « les membres

33. http://www.produitenbretagne.bzh/, consulté le 14 août 2014.

34. Jean Laurent BRAS, «La tournée pour l'emploi de Produit en Bretagne », OuestFrance, 15 août 2014.

35. « Produit en Bretagne. L'association fait école en Catalogne », Le Télégramme, 12 juillet 2014.

36. S. STERVINOU et S. LÊ, op. cit., p. 176-177.

37. S. StervinOU et S. LÊ, ibid., p. 172. 
historiques ont eu du mal à accepter les nouveaux arrivants des départements les plus à l'est », soupçonnés de moindres convictions régionales.

Sur ce point, la réalité est quelque peu différente ${ }^{38}$. Il faut savoir que l'initiative de la création de «Certifié Bretagne ${ }^{39}$ » (document 5) revient au départ à deux acteurs majeurs de la vie régionale, l'un dans le secteur des médias puisqu'il s'agit du quotidien Le Télégramme, l'autre dans le domaine bancaire, en l'occurrence le Crédit Mutuel de Bretagne. À l'exemple d'actions menées au Québec dans le même esprit, les deux partenaires, assez vite rejoints par quelques entreprises de l'agroalimentaire breton et de la grande distribution, se proposent de déclencher un "achat citoyen breton» de la part des consommateurs locaux, en leur expliquant que l'emploi régional est fortement tributaire de leurs achats. C'est aussi la raison pour laquelle ils cherchent à élargir tant la zone géographique de leur intervention que le panel des entreprises concernées.

Ce faisant, ils craignent effectivement une moindre motivation « régionale» de la part des entreprises d'Ille-et-Vilaine dans la mesure où elles ne voudraient pas « se marquer » comme étant trop bretonnes, mais pour des raisons inverses de celles indiquées par Stervinou et Lê : la crainte des promoteurs de PeB était en effet que « ça ne prenne pas, car Rennes est tourné vers Paris et le sentiment d'appartenance à la Bretagne y était moindre. Il était plus facile de coopter le sel de Guérande ${ }^{40} »$. Jean Bannier rapporte également que «dans les textes d'origine, il n'est nullement question de langue bretonne, mais ce n'était pas un choix délibéré, réfléchi ni argumenté. Personne n'y avait pensé ». Ce n'est pas surprenant : en 1993, la perception des fondateurs de PeB à l'égard de la langue régionale est

38. La genèse de PeB est surtout présentée ici comme contribution à l'histoire de l'émergence de cette structure, mais aussi comme un éclairage sur le contexte économique et sociolinguistique de l'époque, au regard de l'approche de la question de la langue bretonne par les milieux économiques. Lire en annexe le témoignage de Jean Bannier, alors directeur de la communication du CMB, 20 août 2014.

39. C'est la première dénomination de la structure, informelle au départ. Lire en annexe le témoignage de Jean Bannier.

40. Témoignage téléphonique de Jean Bannier, 20 août 2014. 
tout à fait conforme à celle repérée parmi les chefs d'entreprise à la même époque par les lycéens de Guingamp ${ }^{41}$.

\section{CERTIFIÉ B RETAGNE}

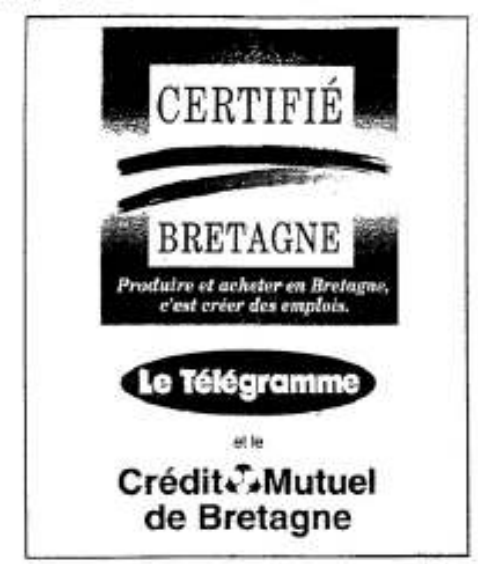

Document 5.

L'article du Télégramme du 8 novembre 1993 annonçant le lancement de l'opération «Certifié Bretagne » qui changera de dénomination quelque temps plus tard pour devenir «Produit en Bretagne».

\section{"Brezhoneg \& business"}

En 1999, la structure décide pour la première fois de prendre en compte l'usage qui se fait du breton en Bretagne, de suivre l'exemple initié les années précédentes par Intermarché et d'éditer à l'occasion de la fête de la saint Yves (devenue fête de la Bretagne) un prospectus publicitaire bilingue que diffusent cette fois toutes les enseignes de la grande distribution dans la région (document 6). Il faut ensuite attendre 2006 et 2007 pour qu'elle prenne l'initiative d'une campagne publicitaire d'envergure avec le slogan breton «Plijadur penn-da-benn », sous-titré « Du plaisir, du début à la fin » [Ravis, tout simplement] dans le métro parisien (document 7).

Depuis les années 1990, s'est donc ouvert un nouveau chantier que ses promoteurs eux-mêmes reconnaissent comme ambitieux et qui ne vise rien moins qu'à présenter la langue bretonne comme « une des richesses dont dépend l'économie régionale ». L'entreprise asso-

41. Voir supra. 


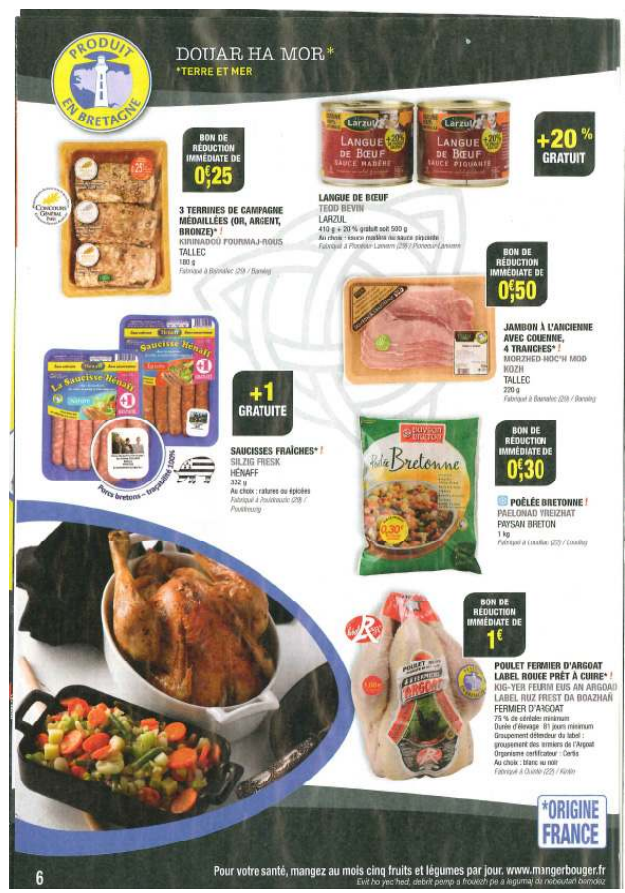

Document 6.

Exemple d'une page intérieure de flyer (prospectus), diffusé par les enseignes de la grande distribution à l'occasion de la fête de la saint Yves ou de la fête de la Bretagne. On remarque que l'identification première du produit est présentée en caractères gras et s'impose au regard, alors que le breton, au-dessous, est en grisé et n'apparaît pas indispensable à la compréhension. Le commerce va droit au but et ne veut pas perdre de clients.

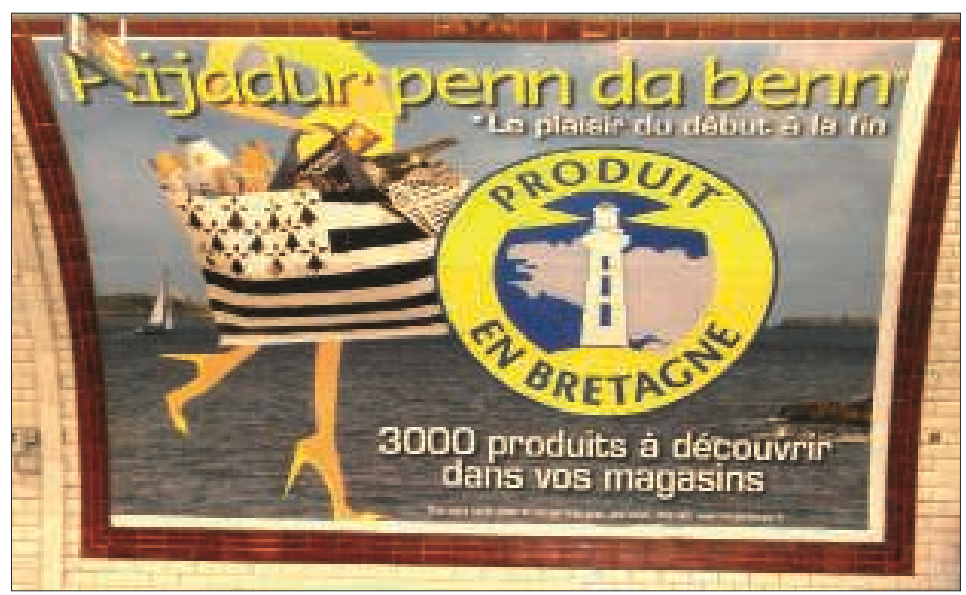

Document 7. La campagne d'affichage « historique » de « Produit en Bretagne » dans le métro, à Paris, en 2007. L'utilisation du breton au haut de l'affiche est effectivement une première, mais le choix de mener cette campagne à Paris et non en Basse-Bretagne est assez symbolique : le breton n'est plus perçu comme langue d'usage, mais comme un outil de promotion. En même temps, l'affiche se présente graphiquement avec une prédominance et une forte visibilité du logo et des textes en français. 
ciative qu'est $\mathrm{PeB}$ selon Wikipedia ${ }^{42}$ apparaît assurément comme le maître d'œuvre de ce chantier. Le numéro spécial que publie la revue $\mathrm{ArMen}^{43}$ en 2012 est conçu à la fois comme un état des lieux et comme un vade-mecum à l'intention des entreprises qui souhaitent s'investir « en faveur de la langue bretonne ». Les contributions réunies dans ce hors-série énoncent les postulats qui sous-tendent le projet « brezhoneg \& business » et décrivent les outils qu'il met en œuvre, ainsi que les obstacles qu'il peut rencontrer.

De toute évidence, les préoccupations initiales, en 1993, sont uniquement d'ordre économique, et ce n'est que cinq ans plus tard, en 1998, que sont décernés pour la première fois les prix « culture et création » de $\mathrm{PeB}$, après que les entreprises culturelles purent adhérer à l'association. Comment en est-elle venue à intégrer une dimension culturelle dans son projet? Sur son site, est formulé le postulat selon lequel il convient de « lier la culture bretonne à l'économie », énoncé à l'occasion de la signature de la charte «Ya d'ar brezhoneg » [Oui au breton]. C'est surtout dans l'éditorial d' $\mathrm{ArMen}^{44}$ qu'est fortement exprimé le concept de « réconcilier l'économie et la culture », jugé d'autant plus nécessaire sans doute qu'une autre contribution dans la même revue fait précisément le constat « qu'il existe une coupure nette entre économie et culture dans l'esprit du public ${ }^{45} »$.

Ce qui est certain, c'est que le monde de la culture ne peut guère s'abstraire des contingences budgétaires ou économiques. Par contre, l'affirmation selon laquelle la culture serait « une condition préalable aux échanges ${ }^{46}$ » surprend dans la mesure où l'échange

42. https://fr.wikipedia.org/wiki/Produit_en_Bretagne, consulté le 14 août 2014.

43. Comme tous les numéros d'ArMen, celui-ci signale Jacques Fitamant comme directeur de la publication et Yann Rivallain comme étant alors le rédacteur en chef. Il ne fait aucun doute qu'il a été élaboré - et possiblement cofinancé - en étroite collaboration avec PeB. L'essentiel de ce numéro spécial est d'ailleurs constitué d'une reprise d'articles de Malo Bouëssel du Bourg, directeur général de $\mathrm{PeB}$, déjà parus en version bretonne dans les précédentes livraisons de la revue, accompagnés cette fois d'une traduction française. ArMen publie toujours dans chacun de ses numéro la chronique en langue bretonne de M. Bouëssel du Bourg, "Ar bed e don ma faner » [Le monde dans mon panier].

44. Y. Rivallain, op. cit., p. 5.

45. Malo BouËSSEL DU BouRG, « La langue bretonne et l'économie », ArMen, op. cit., p. 21.

46. M. BOUËSSEL DU BOURG, ibid. 
est, en sciences économiques, l'acte par lequel un bien est fourni en contrepartie d'un autre : l'histoire depuis l'Antiquité et la vie quotidienne toujours aujourd'hui fourmillent d'exemples d'échanges marchands entre peuples et individus ne partageant ni la même culture ni la même langue. Dans le cas précis de PeB, la donne est quelque peu différente cependant, non que la culture soit réellement « un préalable » à l'échange, mais parce que les différentes entreprises qui en sont membres, qu'elles relèvent du secteur culturel ou de celui de l'agroalimentaire, se confortent en se côtoyant : comme l'écrit Roseline Le Squère, « chacun y voit un intérêt valorisant pour son produit, son entreprise, son institution ${ }^{47} »$.

\section{Langue et identité}

Définissant PeB comme une marque ombrelle, la chercheure observe astucieusement que les entreprises culturelles trouvent grâce à elle une porte d'entrée pour leurs livres et $\mathrm{CD}$ dans les grandes surfaces auxquelles elles n'avaient pas aisément accès auparavant, et qu'elles donnent en même temps du crédit à tous les autres produits vendus sous le même label : « les éléments culturels [...] bénéficient de représentations positives de la part des consommateurs, dans le sens où ces produits équivalent à un achat "noble", à la différence d'un produit alimentaire qui paraît plus banal, plus commun [...]. Le consommateur n'achète pas qu'un produit. Il achète aussi l'assurance d'une qualité, l'assurance que cela réponde au besoin qu'il s'était fixé initialement ». En créant « une cohérence » entre tous les types de produits qu'il labellise, qu'ils soient culturels ou non, le logo jaune de $\mathrm{PeB}$ en est venu à une forme de représentation symbolique de la Bretagne.

Cela joue-t-il de la même manière en faveur de la langue ? Georg Kremnitz rappelle que la pratique d'une langue sert deux buts à la fois, d'une part la communication, de l'autre la démarcation : « quelle que soit la variété linguistique dont j'use, je m'en sers à la fois pour communiquer avec d'autres, mais je m'inscris en même temps dans mon ou mes cercles de communication et je me démarque

47. Roseline LE SQUÈRE, «Cultures régionales et développement économique. Le panorama breton ", Langues régionales, cultures et développement, op. cit., p. 214. 
de tous les autres ${ }^{48} »$. Pour ceux qui le connaissent et qui le parlent, le breton sert donc comme toute autre langue à la fois de moyen de communication et de marqueur d'identité. Est-ce parce que le nombre de ses locuteurs est en diminution qu' ArMen insiste plus sur sa fonction démarcative que sur sa fonction de communication ?

Malo Bouëssel du Bourg, le directeur de PeB, le répète dans ses articles: " la langue est une des composantes les plus profondes de l'identité bretonne ${ }^{49} »$. Les promoteurs de l'utilisation du breton dans le monde économique s'interrogent cependant eux-mêmes sur l'intérêt qu'elle représente : "pourquoi donc faire usage du breton sur un packaging ? » L'élément de réponse avancé est le suivant : « le breton est devenu une sorte de bannière derrière laquelle se rassemble toute la population ${ }^{50}$, quand bien même la plupart des Bretons ne le parlent pas encore ${ }^{51} »$. La langue de bois étant « un langage figé, stéréotypé, reflétant une position dogmatique ou cherchant à éluder une réalité embarrassante ${ }^{52} »$, on pourrait en avoir là une belle illustration. Sur la base du dernier sondage réalisé sur la pratique du breton ${ }^{53}$, l'auteur fait certes état de 200000 locuteurs environ sur une population de 4,5 millions d'habitants dans la Bretagne historique et s'appuie sur le fait que près de $90 \%$ des Bretons sont d'avis qu'il faut conserver le breton. Mais il méconnaît que le pourcentage de ceux qui jugent indispensable ou utile de le savoir est inférieur à ceux qui ont une opinion contraire et que le taux de ceux qui se déclarent attachés à la langue bretonne reste certes élevé, à $56 \%$, mais qu'il est en nette diminution par rapport à un précédent sondage. Mais quand on sait que le nombre de bretonnants est en diminution rapide depuis plusieurs dizaines d'années et que celui des nouveaux brittophones

48. Georg KREMnITZ, «Introduction générale », Histoire sociale des langues de France, Georg Kremnitz (dir.), Rennes, Presses universitaires de Rennes, 2013, p. 27.

49. M. BOUËSSEL DU BOURG, op. cit., p. 21.

50. Lorsque sont organisées des manifestations en faveur de la langue, par exemple pour réclamer la ratification de la Charte européenne des langues régionales ou minoritaires à Quimper le 31 mars 2012, elles peuvent rassembler jusqu'à 10000 personnes et plus.

51. M. BOUËSSEL DU BOURG, op cit., p. 23.

52. Antidote 8, correcteur, dictionnaires, guides, Montréal, Druide informatique, 2014.

53. F. BROUDIC, Parler breton au XXIe siècle..., op. cit. 
ne parvient pas à compenser la disparition des bretonnants plus âgés, laisser entendre que « la plupart des Bretons » pourraient le parler à brève échéance alors qu'ils ne le font «pas encore » est quelque peu amphigourique.

\section{Un argumentaire positivant}

Diverses autres assertions dans l'air du temps sont présentées comme des évidences qui ne peuvent aisément prêter à discussion :

- «L'esprit du breton est assez voisin de l'esprit bio. C'est de qualité de vie qu'il est question... ${ }^{54}$.»

- Introduire la langue bretonne dans l'entreprise ? « Une façon de penser qui s'accorde aux principes du développement durable ${ }^{55} . »$

- «Le mot Breizh, bien compris des non bretonnants, apporte [...] une nuance moderne et engagée ${ }^{56}$. »

- "Le breton est devenu quelque chose de moderne. Si le breton est moderne, l'entreprise qui l'utilise sera moderne. Si la langue déborde d'énergie, le magasin profitera de cette énergie ${ }^{57}$. »

Mais peuvent-elles séduire, si on y réfléchit bien, au-delà de ceux qui partagent l'autre terme de l'alternative ? L'un peut, de fait, servir de passerelle vers l'autre : le bio vers le breton, par exemple, ou l'inverse. Mais le spectre ne couvre pas dès lors tous les champs du possible, et si l'un ne suscite pas l'adhésion, l'autre ne le fera pas non plus. Toujours est-il que les qualificatifs associés à la langue bretonne n'ont absolument plus la connotation négative d'une époque révolue. Le tableau suivant est un relevé, selon quatre thématiques, de la terminologie incitative visant à convaincre un chef d'entreprise, qu'il soit bretonnant ou non, de l'intérêt qu'il aurait à user de la langue bretonne dans le cadre de son activité ${ }^{58}$.

54. ArMen, op. cit., p. 33.

55. Ibid., p. 39.

56. Ibid., p. 25.

57. Ibid., p. 33.

58. Ibid. 


\begin{tabular}{|l|l|l|l|}
\hline Affectif & Valeurs & Langue & Territoire \\
\hline Atmosphère différente & Amitié & Contemporaine & Breizh \\
Capital sympathie & Authenticité & Énergie & Proximité \\
Humain & Convivialité & Moderne & \\
Moments de bonheur & Du plus & Ouverte sur le & \\
Plaisir & Fidélité & monde & \\
Potentiel positif & Fierté & & \\
& Lien social & & \\
& Solidarité & & \\
\hline
\end{tabular}

Le relevé n'est sans doute pas exhaustif, et le classement possiblement subjectif. L'argumentaire est, ici, forcément positivant et les thématiques dominantes relèvent de l'affectif ou des valeurs : ne pourrait-il d'ailleurs pas être transposé tel quel à bien d'autres langues, qu'elles soient minoritaires ou pas? Aucun bretonnant ne peut nier, de fait, que l'usage de sa langue peut aisément conduire à un échange chaleureux, sympathique ou convivial avec un interlocuteur de rencontre.

Des réticences ou des blocages transparaissent pourtant. Dès l'éditorial du hors-série assurant la promotion du breton dans le monde économique, Y. Rivallain ${ }^{59}$ reconnaît que « le breton [n'est] que trop rarement employé pour évoquer la vie économique et celle des entreprises, alors que l'avenir de la langue découle évidemment de son utilisation dans l'ensemble des situations de la vie quotidienne, y compris au travail ». Le contraste est frappant, en apparence, entre ces deux affirmations juxtaposées. L'auteur, d'une part, fait lucidement le constat que le breton n'a plus guère fonctionnellement sa place dans le monde de l'économie (ce qu'attestent les résultats du sondage TMO Régions de $2007^{60}$ ), et il est tout à fait conscient d'autre part que, pour ne pas péricliter, il devrait idéalement devenir langue d'usage pour le plus grand nombre aussi bien dans la vie professionnelle que dans la vie quotidienne. Significativement, il ne dit pas 'redevenir', oubliant qu'il en a été ainsi antérieurement, et il n'y a pas si longtemps. L'évidence du projet d'avenir qu'il formule pour la langue bretonne est donc l'exigence d'une reconquête à multiples

59. Y. Rivallain, op. cit., p. 5.

60. Voir infra. 
facettes, celle d'un monde à (re) construire à tout le moins, et celle d'une démarche à laquelle devrait adhérer le plus grand nombre.

«Et pourtant, concède l'un de ses promoteurs, il est bien difficile de développer l'usage du breton dans l'économie ${ }^{61}$. » Il a beau y avoir un consensus « naturel» au sein de PeB en sa faveur, « cela ne veut pas dire que tous les membres [...] sont sensibles à la question linguistique [...]. Cette posture globale ne se traduit pas automatiquement par une motivation de chaque dirigeant à donner une place à la langue bretonne dans son entreprise ${ }^{62} \gg$. La place du breton et de l'image qui lui était attachée paraît avoir fait l'objet, à un moment donné, de débats « approfondis ${ }^{63}$ » au sein de PeB. L'actuel président de l'association, Jacques Bernard, raconte qu' « au début, il n'y avait pas franchement de rejet, mais certains se demandaient à quoi ça pouvait "servir" [...]. [N]ous avons expliqué, hors de tout contexte militant, l'importance de la langue pour la culture bretonne en général. Aujourd'hui tout le monde s'est rallié à l'idée, sans état d'âme ${ }^{64} »$. Le directeur, M. Bouëssel du Bourg, nuance le propos : « a minima, le choix de donner une place à la langue bretonne est compris et partagé ${ }^{65} »$.

\section{Ceux qui apparaissent comme les "précurseurs"}

Si on la lit attentivement, cette restitution feutrée des débats liés à l'usage du breton dans le monde de l'économie témoigne de quelques tensions, autrement dit d'une certaine insécurité linguistique : il n'est pas si facile de reconnaître à une autre langue, régionale en l'occurrence, la même légitimité que celle acquise de longue date et de facto par la langue nationale, voire par l'anglais en tant que langue principale des échanges à l'international, y compris et surtout dans le monde des affaires et du commerce. Dans le cas présent, les premiers concernés sont les chefs d'entreprise, autrement dit les décideurs : leur adhésion à la démarche est-elle aussi forte qu'on pourrait le croire?

61. M. BOUËSSEL DU BOURG, op. cit., p. 21.

62. Y. RivalLAIN, op. cit., p. 12-13.

63. Ibid.

64. Ibid.

65. Ibid. 
Il n'est dès lors pas étonnant qu' $\mathrm{ArMen}^{66}$ considère comme « des pionniers » ceux qui les premiers ont pris la décision d'afficher du breton dans leur supermarché : « chaque fois que des panneaux bilingues sont installés dans un magasin, on découvre des hommes motivés », et il faut l'être, c'est vrai, lorsqu'on se veut innovant. S'ils l'ont été, ce n'est pas en général parce qu'ils sont eux-mêmes locuteurs, mais en raison de leur itinéraire personnel, de leurs convictions ou de la représentation qu'ils se font d'une langue qu'ils ne parlent pas. Trois des cas que présente la revue $\mathrm{ArMen}^{67}$ paraissent à tout le moins emblématiques :

- Georges Prémel-Cadic, considéré comme "le déclencheur» depuis les initiatives qu'il a prises à l'Intermarché de Plouguerneau, explique ainsi ses motivations : « Je fais partie des chaînons manquants : mes parents ne m'ont pas transmis leur langue maternelle. Ma prise de conscience fut tardive, vers 1718 ans, d'abord révoltée, puis constructive [...]. Étant décideur dans mon entreprise, je pouvais agir. Intégrer la langue bretonne dans la vie de tous les jours, dans le commerce, me paraissait fondamental ».

- Le profil d'Hervé Le Goff, directeur du Super U de Keredern, à Brest, est analogue : alors que ses parents savaient le breton, ils ne le lui ont pas appris. Il ne le parle pas, mais le définit comme "sa langue de cœur» et explicite à partir de cette intériorisation les fondements de son engagement : "On ne peut pas se contenter de vendre des petits pois [...]. Ce ne sont plus "les ploucs" qui parlent breton, mais des gens qui ont fait un choix. Par l'intermédiaire du breton, on crée une atmosphère différente et plus humaine dans le magasin. Une enseigne nationale peut aussi être locale ».

- Pour la réouverture après travaux de la FNAC de Rennes, en 2010, la responsable de la communication du magasin, Anne Giumelli, a tout de suite « été séduite par l'idée [que lui avait suggérée un conseiller municipal de la ville, bretonnant] de mettre le breton bien en évidence dans un contexte ultramoderne. C'était quelque chose d'inhabituel pour la 
FNAC [...]. J'ai très rapidement obtenu un avis favorable [de Paris]. Après quoi, j'ai collaboré avec l'Office public de la langue bretonne [...]. Chaque jour, quand je regarde les panneaux, je retrouve ces moments de bonheur ». Mais vous ne le saviez peut-être pas, car ce n'est pas tout : «bilhedoù (billetterie), c'est mignon » (sic).

L'homonymie approximative entre un terme breton purement technique et une expression française de la relation amoureuse devient tout à coup une bonne raison d'afficher du breton dans un grand magasin. Au-delà du clin d'œil, on retrouve ici plusieurs des qualificatifs associés à la langue précédemment relevés, jusqu'au paroxysme associant l'image d'une langue qui n'en avait apparemment pas tout à fait la caractéristique à l'ultramodernité d'une grande enseigne culturelle. Les promoteurs du bilinguisme dans la grande distribution avancent d'autres arguments sur divers registres et tous convergent vers une même finalité ${ }^{68}$ :

- L'intime conviction d'une mission à assumer : «On ne peut pas prendre une partie de notre culture et jeter le reste à la poubelle. C'est notre devoir de donner quelque chose à la langue. Et même si l'on n'est pas capable de parler breton, on peut toujours faire quelque chose et devenir acteur. »

- La pédagogie par l'exemple : le patron d'un centre Leclerc de la périphérie rennaise est persuadé de faire tache d'huile « si un magasin reprend l'idée, puis un autre...».

- Le besoin supposé de retrouver des racines et la recherche d'une compensation par rapport à la mondialisation à laquelle tout un chacun est confronté : "la langue bretonne jouit d'un potentiel positif. Elle apporte de la proximité et de la convivialité aux Bretons autant qu'aux étrangers ».

- L'argument central est cependant celui de l'identité, ce qui explique qu'il est décliné selon de subtiles variations. Pour Jean-Claude Le Gall, ancien dirigeant de Saveol et ancien président de PeB, le breton est « au cœur de l'identité bretonne et de ce qui rend ce pays spécifique ». Georges Prémel-Cabic est convaincu que PeB n'existe que parce que la Bretagne a 
une identité forte, ce dont peu de régions peuvent se prévaloir » et cette identité, explique-t-il, est multiple: géographique, historique et culturelle - la culture étant considérée dans le cas présent comme incluant la dimension linguistique ${ }^{69}$. Selon Malo Bouëssel du Bourg, « la langue est une des composantes les plus profondes de l'identité bretonne ${ }^{70} »$.

\section{Le bénéfice qu'en attendent les entreprises}

Toutes ces belles proclamations que mettent en avant chefs d'entreprise et responsables économiques n'auraient-elles pour finalité que de contribuer au renforcement tautologique de l'identité culturelle et linguistique de la Bretagne ? Elles l'ont probablement, mais ce discours sur l'identité est présenté en l'occurrence comme ayant une qualité supplémentaire susceptible d'intéresser - au sens propre - les catégories professionnelles concernées: l'assurance qu'ils pourront en retirer un bénéfice. Un directeur de supermarché l'exprime très simplement : puisque la langue bretonne est synonyme de proximité et de convivialité, affirme-t-il, "il ne tient qu'à nous d'en récolter les fruits ${ }^{71} \gg$.

Le directeur de PeB est encore plus direct: «l'identité de la Bretagne fait vendre et la langue est un marqueur identitaire efficace $^{72}$ », affirme Malo Bouëssel du Bourg. Il assure, exemples à l'appui, qu' " augmenter les ventes et promouvoir la langue [bretonne] ne s'opposent pas » : il s'agit très concrètement d'user du breton pour vendre du café, de l'eau de source, des yaourts, de la bière ou du whisky par différents procédés, que ce soit une appellation de marque à consonance bretonne et la mise en évidence de quelques mots ou quelques lignes de breton sur l'emballage. C'est en quelque sorte mettre l'identité de la Bretagne et la langue bretonne au service du marketing. Le tout est de savoir dans quelle mesure la présence de breton sur un produit déclenche l'acte d'achat: si l'un ne s'oppose pas à l'autre en soi, il ne garantit sans doute pas non plus un automa-

69. Voir supra.

70. ArMen, op. cit., p. 12, 19, 21.

71. ArMen, op. cit., p. 35.

72. Ibid., p. 14. 
tisme de la part de l'acheteur potentiel. Sinon, pourquoi donc toutes les marques n'ont-elles pas encore adhéré à la démarche ?

De fait, et de l'avis même des promoteurs d'un tel choix entrepreneurial, les retombées sonnantes et trébuchantes ne sont pas d'emblée au rendez-vous. Les chefs d'entreprise doivent cependant se convaincre d'une promesse de retour sur investissement : «la langue bretonne, dit une élue régionale, saura sans nul doute rendre à l'entreprise ce que l'entreprise aura su lui donner ${ }^{73} \gg$. En attendant, Steven Guéguéniat, patron de PME à Brest, reconnaît franchement qu'en faire usage « cela ne rapporte pas d'argent : il est plus facile de créer l'esprit d'équipe avec des places de foot qu'avec le breton ${ }^{74}$ ! » Denez Pichon, lui aussi bretonnant et convaincu de l'intérêt de parler la langue, a créé Askorn Medical, une société spécialisée dans le développement de systèmes médicaux et d'implants orthopédiques à Cesson-Sévigné, et confirme : "cela suscite sans doute de la notoriété, mais pas vraiment de revenu ${ }^{75} »$. Que reste-t-il alors du bénéfice escompté ?

Le directeur de PeB l'exprime d'une façon pragmatique et directement compréhensible : «[...] les dividendes de la culture ne se perçoivent pas à court terme, écrit-il. Il s'agit d'un investissement dont le retour ne s'évalue pas au moyen d'un taux, mais à l'aune de l'engagement des collaborateurs et par les valeurs et le plaisir qu'ils vont ainsi partager ${ }^{76} \gg$. Explicitant sa pensée, il précise ailleurs que «la mesure de [1'] utilité pratique [de la langue bretonne] doit être complétée par [celle] de la valeur ajoutée immatérielle, sociale et sociétale ${ }^{77} \gg$. Faisant référence à un commerce de boulangerie en zone rurale à Saint-Cadou, en Sizun (Finistère), où les heures d'ouverture et les étiquettes sont entièrement bilingues et où les clients sont accueillis en breton, M. Bouëssel du Bourg admet que « la plupart des entreprises ne sont pas encore prêtes à aller aussi loin [...]. L'usage de la langue bretonne permet de nouer de nouveaux liens

73. Ibid., p. 9.

74. Ibid., p. 39.

75. Traduit du breton. Denez Pichon, « Re verr ar vuhez evit chom displijet re bell » [La vie est trop brève pour rester trop longtemps contrarié], $\mathrm{Ya} !, \mathrm{n}^{\circ} 485$, 26 septembre 2014, p. 12.

76. ArMen, op. cit., p. 21.

77. Ibid., p. 13, 21. 
entre l'économie, les habitants et le territoire. Le breton augmente le capital humain et social de l'entreprise ${ }^{78} \gg$.

Esquissons, à ce stade, une synthèse à partir du discours de PeB et de l'argumentaire des chefs d'entreprise: l'usage de la langue bretonne au sein de l'entreprise ne se traduit pas d'emblée en dividendes et la valeur ajoutée qu'elle peut en retirer se situe essentiellement au niveau de l'immatériel, qu'il s'agisse de la qualité des relations qu'elle parvient à établir avec son personnel (en interne) ou du lien social qu'elle contribue à créer avec son environnement (en externe). Ce constat induit une question toute simple: dans quelle mesure un chef d'entreprise, s'il est bretonnant et surtout s'il ne l'est pas, a-t-il intérêt à s'impliquer pour introduire ou développer l'usage du breton pour son fonctionnement si le plus qu'il peut en retirer ne peut être financier et qu'il n'est dès lors que d'ordre qualitatif?

Pour Denez Pichon, le breton présente un intérêt majeur : il lui permet de différencier son entreprise Askorn Médical par rapport aux autres. Il ne lui apporte pas vraiment de clientèle supplémentaire, mais il a l'intérêt de susciter l'adhésion du personnel en interne, de créer du lien avec ses partenaires et d'attirer l'attention de ses clients à l'étranger ${ }^{79}$. À la tête de l'imprimerie brestoise Ouestelio, Steven Guéguéniat a réfléchi à un business model qui tient compte de ce questionnement et du parti qu'il peut tirer d'un usage convaincant et modulable de la langue bretonne. Il ne l'impose pas, mais l'utilise partiellement dans le cadre du fonctionnement de son entreprise ainsi que pour la publicité, si bien que la langue est perçue comme étant chez lui « bien autre chose qu'une lubie [...]. Nous sommes les seuls à faire cet usage du breton dans la région ${ }^{80} »$. Il transforme donc sa maîtrise de la langue en atout et en tire argument pour accroître sa notoriété et se positionner face à la concurrence. Dans son cas, le retour sur investissement passe dans un premier temps par une phase de développement de sa clientèle. Ce retour ne sera pas de même nature dans toutes les PME, ni a fortiori dans des entreprises de la grande ou de la moyenne distribution. Cela dépend à la fois de la nature de l'entreprise et de sa clientèle.

78. Ibid., p. 41.

79. D. PICHON, op. cit.

80. ArMen, op. cit., p. 39. 


\section{Les modalités de présence du breton dans le commerce}

Hypers et supermarchés visent en permanence à capter la plus grande part de marché - autrement dit de clientèle - dans leur zone de chalandise. Cette captation se joue sur de multiples paramètres, dont le moindre n'est pas la guerre des prix que se livrent les différentes enseignes. Ces dernières se disputent aussi sur une certaine idée de la proximité qui les conduit à mettre en avant des lignes de produits (ainsi « Nos régions ont du talent » chez Leclerc), des produits considérés comme typiquement régionaux (biscuiterie, conserves ou objets symboliques, par exemple) aux périodes d'afflux des touristes, ou encore les relations privilégiées qu'elles établissent contractuellement avec des entreprises ou des producteurs locaux ${ }^{81}$. Cette politique va dans certains cas jusqu'à l'intégration de pans entiers de filières de production, que ce soit dans l'abattage ou dans la pêche (notamment chez Intermarché et Leclerc). La proximité n'est assurément pas le seul élément qui guide cette politique, mais plusieurs enseignes nationales tiennent aujourd'hui à apparaître comme étant locales également.

La présence du breton dans le commerce et les affaires relèvet-elle de la même démarche? Elle ne se situe pas sur le même registre en tout cas, puisqu'elle ne représente pas une production identifiable en tant que telle. Elle se décline dès lors selon diverses modalités (document 8) :

- Des appellations de marques connues de longue date : Traou mad (les célèbres biscuits de Pont-Aven), Krampouez ar vro (de la crêperie Lamer à Lampaul-Gumillau), Krampouz (société de fabrication de crêpières créée par Jean-Marie Bosser en 1949), Rosko (transports à Saint-Pol-de-Léon).

- Des marques de création plus récente : Breizh Cola, Carabreizh (caramels au beurre salé à Belle-Île-en-Mer), Ker Ronan (yaourts fabriqués à Rohan dans le Morbihan), Eddu

81. Les initiatives que prennent les enseignes elles-mêmes pour afficher leur proximité avec leur clientèle s'apparentent à celles de PeB, si ce n'est que dans un cas les initiateurs sont les distributeurs eux-mêmes, alors que dans l'autre ce sont en quelque sorte les producteurs qui sont à l'origine. La démarche, pour être parallèle, n'est pas strictement identique, mais l'objectif affiché est le même, puisqu'il s'agit de se positionner en faveur d'une forme de « relocalisation » des produits et de contribuer à l'emploi local ou régional. 


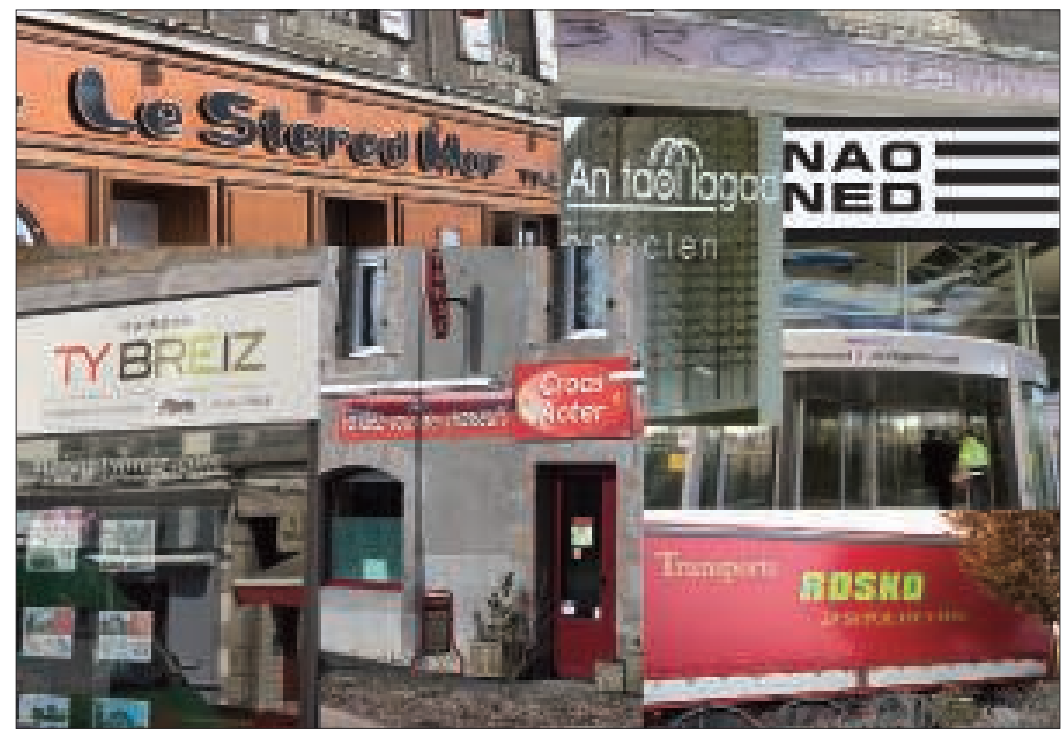

Document 8. Sur la photo : le Stered mor, café à Cléder ; les maisons Ty Breiz à Pontivy : le magasin de tricot Brochenn à Landerneau ; l'opticien An taol lagad à Pleyber-Christ ; le logo de Naoned, une nouvelle marque de lunettes conçues et fabriquées à Nantes ; l'entrée de l'aéroport de Brest; le café Croas Aoter à Plourin-Ploudalmézeau ; un camion des transports Rosko, de Saint-Pol-de-Léon. On conviendra que le choix d'An taol lagad (Le coup d'œil) par un opticien est... bien vu, d'autant qu'il reprend la dénomination du journal en breton de France 3 Iroise. Le logo de Naoned peut se décrypter comme signifiant plus qu'une implantation géographique, comme une conviction aussi. Croas Aoter indique un lieu topographique connu comme tel, en complément de Rendez-vous des chasseurs, et il est rédigé «à l'ancienne», sans autre considération ni intervention extérieure.

(whisky de Plomelin), Breizh Konfitur (à Pleuven), D'istribilh (bières à Plouider).

- Des noms de produits : Breizhpresso, Breizh Soupe, Breizh gaufrette, Breizh Gwin Gwenn, Erminig sukret, Galleg (un whisky de la distillerie Warengheim à Lannion), Breizh kontell (une collection régionale de L'Atelier des couteaux à Tulle, en Corrèze). À Pluguffan (Finistère), l'une des 400 références des cafés Tanneau est au nom de Kan an avel.

- Des enseignes de magasins et de commerces divers et variés : Biogastell (à Plougastel-Daoulas), Mat Kalon (en gare de Rennes), Alré Bio (à Auray), Ar Vro (ambulances, taxi, librairie, restaurants), Croas Aoter (café à Plourin-Ploudalmézeau) Rien que dans le Finistère, on peut relever dans l'annuaire 
environ 70 noms de crêperies ayant un dénominatif breton : $A r$ chupenn, Ar Saozon, Breiz-Izel, An Teuzar, Ti Breiz... (document 9). À Quéven (Morbihan), Le Marché du bon goût se présente en même temps comme "Marc'had ar blas mat». Inutile de préciser que certaines de ces enseignes sont éphémères, quand d'autres sont plus durables : près des halles Saint-Louis de Brest, le bistro Lak e-barzh n'existe plus sous ce nom.

- Le packaging ${ }^{82}$ : l'eau de source Brocéliande s'annonce « hep nitrat» [sans nitrate] quand l'Isabelle promet « Gant Isabelle ni yelo pell » [Aller loin avec Isabelle]. Les bières Britt s'autoproclament «Bier brudet euz Breizh» [Bière réputée de Bretagne]. Les yaourts Ker Ronan impriment un proverbe breton sur leur emballage. Le lait ribot Bridel affichait discrètement son origine bretonne en écrivant «laez ribot $\gg$ en tout petits caractères sur l'étiquette ${ }^{83}$.

- Les prospectus bilingues de $\mathrm{PeB}$, diffusés chaque année à l'occasion de la Fête de la Bretagne, à deux millions d'exemplaires dans les cinq départements de la Bretagne historique ${ }^{84}$.

- La publicité : depuis sa première campagne bilingue dans le métro parisien, évoquée par ailleurs, $\mathrm{PeB}$ en a mené plusieurs autres, à Paris et en Bretagne, sur le même principe d'un slogan en breton en gros caractères, sous-titré en quelque sorte en français (document 10). L'association a par ailleurs produit et mis en ligne un film de 13' qui se veut tonique pour la promotion du breton dans le monde de l'économie et présente le témoignage de chefs d'entreprise et d'acteurs économiques impliqués ${ }^{85}$.

82. Pour sa part, le directeur de PeB a repéré, écrit-il, « un peu de breton sur quelques emballages. Assez rarement, il faut bien l'avouer, [mais] bon an mal an, une bonne centaine de produits sur lesquels fleurit la langue du pays ", ArMen, op. cit., p.23. Quand on imagine les centaines ou les milliers de produits qui doivent se fabriquer journellement en Bretagne, cela paraît assez peu en effet.

83. Ce lait ribot n'est pas référencé sur le site http://www.lactalis-international.com/ à la date du 12 octobre 2014 .

84. ArMen, op. cit., p. 19-21.

85. Langue bretonne et entreprise. Brezhoneg hag embregerezh. Production : Label production. Réalisation : Jacques Bernard et Mickaël Ragot, 2013, 13 minutes. 


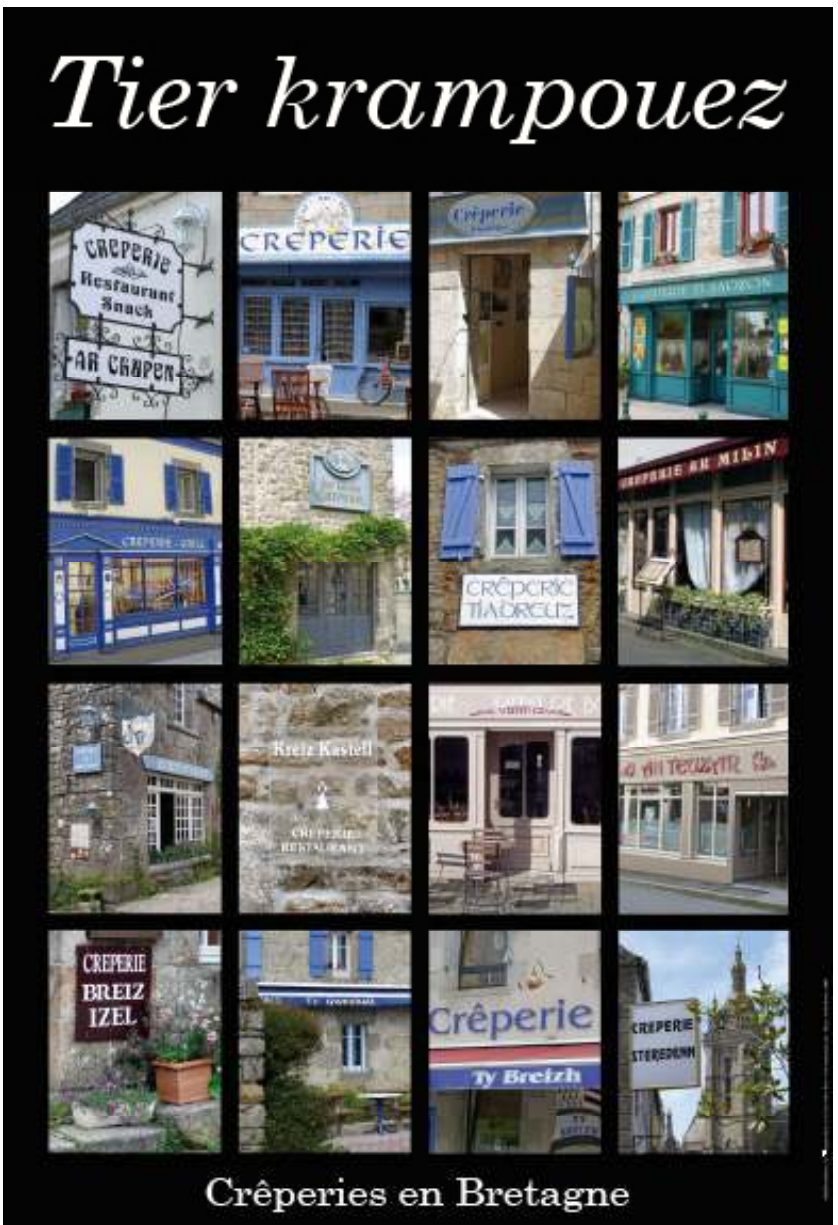

Document 9. Poster conçu par les éditions Emgleo Breiz, présentant 16 enseignes de crêperies en breton. $Y$ aurait-il une association d'idées entre «crêperie» et « langue bretonne » ? Ce n'est pas sûr, car celles qui ont opté pour une appellation en français sont bien plus nombreuses... Si une enseigne en breton s'adresse aux touristes - qui n'en comprennent généralement pas le sens -, elle peut apparaître comme un gage d'authenticité. Pour les locaux aussi, mais il n'est pas certain que tous en ont la compréhension pour autant. De toute façon, le mot « crêperie » est généralement associé à la dénomination de l'enseigne et ne saurait susciter d'ambiguïté.

À voir en ligne sur Dailymotion ou sur le site de PeB : https://www.dailymotion. com/video/x17bq73. 
- Une signalisation bilingue à l'entrée ou à l'intérieur de supermarchés et autres commerces, mais aussi de bâtiments publics. Parmi ces derniers, on peut signaler de simples «Degemer mad» (Bienvenue) à l'aéroport de Brest et de multiples panneaux affichant les deux langues dans les gares de Landerneau ou de Carhaix, par exemple. L'Intermarché de Louargat (Côtes d'Armor) renseigne le nom breton de la commune sur sa façade et celui des rayonnages dans les deux langues. Le Super U du quartier de Keredern à Brest souhaite à la fois « Degemer mat » et « Bienvenue » à sa clientèle et vise à l'orienter par de grands panneaux exclusivement en breton (comme «Ar baraer», le boulanger, ou «Ar pesker», le pêcheur) peints sur les murs et par des panneaux bilingues, plus petits, en bout de rayons.

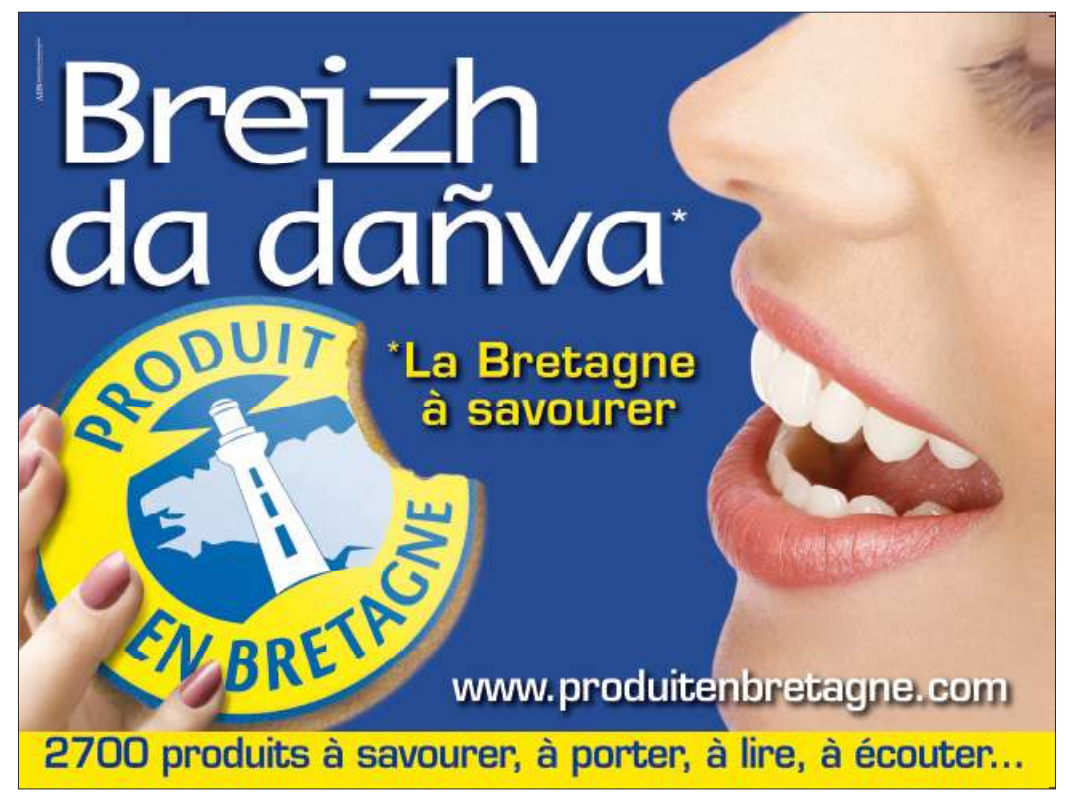

Document 10. Autre campagne publicitaire de « Produit en Bretagne » en 2009. Cette fois, le texte breton est mis en valeur, l'affiche étant conçue pour que celui qui ne le connaît pas en saisisse rapidement le sens. La campagne a été menée à nouveau dans le métro parisien, mais aussi en Bretagne. 
Selon les promoteurs du breton dans la grande distribution, il existe aujourd'hui une demande sociale émanant de parents dont les enfants suivent leur scolarité dans les filières bilingues et que les responsables d'enseignes ou de commerces cherchent à satisfaire, et c'est à leur intention qu'ils entreprennent de bretonniser en quelque sorte l'affichage dans leur entreprise. Si une telle initiative ne cible spécifiquement qu'une population somme toute numériquement restreinte, elle n'apparaît pas dissuasive pour l'ensemble de la clientèle : «rares » sont ceux à qui cela ne plaît pas, dit l'un ; «c'était demandé par les clients », affirme l'autre. À l'Intermarché de Plouguerneau, les réactions ont toujours été positives et ont suscité un sentiment de fierté : d'après son ancien directeur, « cette singularité nous a amené un capital sympathie important ${ }^{86}$ ». Même à Vern-surSeiche (Ille-et-Vilaine), " une part importante » des consommateurs - très exactement $44 \%$ - «plébiscite» la présence du breton sur l'emballage des produits ${ }^{87}$. M. Bouëssel du Bourg estime non sans raison que même les non bretonnants comprennent généralement le mot Breizh, le nom breton de la Bretagne. Il lui semble que lorsque la nature du produit est évidente « nul ne saurait être perturbé ${ }^{88}$ » par un nom de marque breton.

\section{L'économie et l'emploi}

$\mathrm{Au}$ bout du compte, l'emploi de la langue bretonne dans le monde des affaires est-il donc anecdotique ou répond-il à un vrai besoin ? C'est M. Bouëssel du Bourg lui-même qui formule la problématique en ces termes dans l'une de ses contributions à la revue ArMen, qu'il prolonge ingénument - tout en sachant que tous les bretonnants savent aussi le français - par une autre interrogation : «pourquoi [...] n'y aurait-il pas une place pour le breton dans l'économie $^{89}$ ? ». Certes. Et il en a une, mais elle ne paraît pas d'évidence. Quelques repères chiffrés s'imposent.

Selon le dernier sondage TMO Régions, son utilisation sur le lieu de travail est le plus souvent occasionnelle, que ce soit entre

86. ArMen, op. cit., p. 11-15.

87. Ibid., p. 27.

88. Ibid., p. 25.

89. Ibid., p. 19. 
collègues ou dans le cadre d'une relation de subordination. Sur 172000 locuteurs en Basse-Bretagne, 10\% affirment avoir des échanges en breton au travail, mais seul $1 \%$ dit le parler toujours entre collègues quand $4 \%$ le font souvent. Par ailleurs, il n'y a que $2 \%$ des bretonnants à l'utiliser toujours ou souvent dans le cadre des échanges entre employeur et salariés. Dans les banques aussi, la pratique du breton est occasionnelle, puisque seuls $4 \%$ des bretonnants s'expriment en leur langue dans une agence.

Tous les bretonnants ne travaillent pourtant pas sur des postes qui exigent la connaissance de la langue. En 2007, le nombre d'actifs bretonnants était estimé à 42500 personnes, bien moins qu'il n'y en avait dans la seconde moitié du $\mathrm{XX}^{\mathrm{e}}$ siècle assurément. Ces données reflètent en réalité une double tendance que rapportent toutes les enquêtes effectuées depuis les années 1970 : d'une part, le breton est toujours et avant tout la langue de l'amitié, de la convivialité et de la proximité ; son usage concret par les locuteurs eux-mêmes est en régression dans la plupart des registres ${ }^{90}$.

La nouveauté de la période récente est qu'il a lui aussi désormais accès à de nouveaux domaines comme l'éducation, les médias dont internet et certains aspects de la vie publique. Il est par ailleurs diverses fonctions qui contribuent à sa consolidation : avec environ 1300 postes $\mathrm{ETP}^{91}$, il n'y avait jamais eu jusqu'à présent autant d'emplois nécessitant une plus ou moins bonne maîtrise de la langue bretonne $^{92}$. Il convient de prêter attention à leur répartition :

- Plus de 1000 postes, soit environ 80\%, se situent dans le secteur de l'éducation ou de l'enseignement, en progression de plus de $50 \%$ en six ans. Les PE (professeurs des écoles) et les PLC (professeurs des lycées et collèges) en constituent les plus gros bataillons, avec près de 800 postes à eux seuls. Les autres sont des postes d'assistants maternels et d'animateurs, de formateurs pour adultes et d'enseignants et chercheurs du supérieur.

- Les autres professions les mieux représentées concernent la

90. F. BROUDIC, Parler breton au XXIe siècle, op. cit.

91. Équivalent temps plein.

92. Office public de la langue bretonne, La langue bretonne dans le marché du travail en 2012, 2013, http://www.fr.opab-oplb.org, consulté le 13 octobre 2014. 
gestion de structures associatives, les médias et la culture, soit un cumul d'environ 210 postes, correspondant à $16 \%$ de l'ensemble.

- Les autres emplois se localisent dans l'administration publique $(1,5 \%)$ et dans le secteur de la santé et des services à la personne $(0,9 \%)$. Le secteur de l'industrie, de l'artisanat et du commerce affiche un total de 18 postes, soit $1,4 \%$ des emplois liés à la lange bretonne ${ }^{93}$.

L'organisme de formation Stumdi mène deux fois par an une enquête sur le devenir de ses stagiaires ayant suivi une formation longue d'apprentissage du breton. Parmi ceux de 2011, sur 41 réponses, 32 signalent avoir obtenu un poste bilingue à l'issue de leur formation longue en breton, ce qui est gratifiant. Mais $85 \%$ des postes bilingues obtenus l'ont été dans le secteur scolaire surtout, ainsi que dans celui de la culture et de l'édition. Les autres ont été obtenus, à hauteur de $11 \%$, dans le social, et pour $5 \%$ des stagiaires en agriculture. L'Office public de la langue bretonne avance pour sa part le chiffre de 714 organismes du secteur privé ayant signé la charte « Ya d'ar brezhoneg » [Oui au breton] : parmi eux on dénombre 70 sociétés de services, 46 entreprises, 31 grandes surfaces ou commerces alimentaires, 30 autres commerces, 18 sociétés de graphisme, de la publicité et de l'impression, etc. ${ }^{94}$. Une étude sur le devenir des anciens lycéens de Diwan met en évidence que $30 \%$ d'entre eux ont passé le baccalauréat ES (économique et social), soit presque autant que la moyenne française : seuls $9 \%$ ont fait ou font ensuite des études supérieures en sciences économiques ou en AES (administration économique et sociale). Parmi ceux qui sont entrés sur le marché du travail, le groupe le plus important est celui de l'enseignement, suivi de ceux de la santé, des arts et spectacle et de la culture ${ }^{95}$.

93. Les auteurs de l'étude précisent que le chiffre avancé pourrait être sous-estimé, et ce n'est pas improbable.

94. Parmi les 714 organismes du secteur dit privé, signataires de la charte, tous ne relèvent pas à proprement parler du monde de l'économie, puisqu'on y repère aussi de multiples associations du monde culturel ou sportif, des artistes, des médias, etc. Site www.fr.opab-oplb.org/, consulté le 13 octobre 2014.

95. Office public de la langue bretonne, Enquête sur les anciens lycéens de Diwan 2012, http://www.fr.opab-oplb.org, consulté le 13 octobre 2014. 
Au-delà de toutes ces données, de nature différente au demeurant, peut-on savoir quel usage concret de la langue induit cet intérêt pour le breton pour le fonctionnement de l'entreprise ? Certes, des petites entreprises du secteur culturel, telles que Dizale (doublage de films), Kalanna (production de films) ou Stumdi (formation continue) « vivent $[\ldots]$ entièrement pour et par le breton », selon l'expression de Yann Rivallain ${ }^{96}$. Au-delà de ce périmètre, cela semble moins évident. Sachant que plusieurs directeurs de supermarché reconnaissent que le breton est leur langue de cœur et qu'ils ne la parlent pas eux-mêmes, j'ai sollicité le témoignage de deux d'entre eux : ils m'ont expliqué qu'aucun de leurs salariés ne le connaît davantage.

J'ai également souhaité comprendre le fonctionnement de PME à forte notoriété et connues pour leur empathie à l'égard de la langue bretonne. La bière Coreff est une de ces entreprises qu'on ne présente plus depuis qu'elle a été lancée à Morlaix en 1985. Aujourd'hui installée à Carhaix, la brasserie compte une vingtaine de salariés, dont plusieurs ont appris le breton. Le directeur, Mathieu Breton, admet que la langue n'a pas d'usage fonctionnel au sein de la PME. Mais il tient à ce qu'elle soit présente sur les étiquettes des bouteilles qui sortent de la brasserie, dans le musée qui la jouxte et sur le site internet. Comme il veut être certain de la qualité du breton qu'il fera imprimer, il fait donc appel aux prestations de l'Office public de la langue bretonne pour les traductions dont il a besoin ${ }^{97}$.

À Brest, Steven Guéguéniat, également patron de PME, luimême bretonnant et fortement motivé, compte deux autres locuteurs parmi les 19 collaborateurs de l'imprimerie Ouestélio. S'il négocie volontiers avec sa clientèle directement en breton à partir du moment où il l'a identifiée comme pouvant s'exprimer dans la langue, il n'en est pas exactement de même pour ce qui est des relations internes au sein de l'entreprise, pour plusieurs raisons. La première est que les collaborateurs bretonnants n'ont peut-être pas une réelle capacité à s'exprimer précisément en breton concernant leur métier. Le second point sur lequel $\mathrm{S}$. Guéguéniat n'hésite pas à attirer l'attention tient à la qualité des relations sociales dans l'entreprise : il faut surtout éviter, précise-t-il, que ne se créent des barrières au sein du personnel

96. Y. Rivallain, op. cit., p. 13.

97. Entretien téléphonique du 13 octobre 2014. 
et que se constituent deux catégories sur la base d'échanges exclusifs en breton qui donneraient l'impression aux non-locuteurs (en l'occurrence la majorité) que les informations fonctionnelles ne sont pas partagées par tous. Le PDG définit là un autre préalable relatif au recrutement : il est convaincu qu'il doit se faire prioritairement sur le critère de la compétence professionnelle et non privilégier celui de la maîtrise du breton, comme on pourrait être tenté de le faire. Mais il regrette de ne pas recevoir de CV de bretonnants en fin de formation. Par contre, s'il est un point dont tous les collaborateurs de Ouestélio ont bien conscience et qui les motive désormais, c'est qu'une part significative de leur rémunération provient du chiffre d'affaires que réalise l'entreprise par l'entremise du breton, puisqu'il s'élève à $30 \%$ de celui du département édition et à près de $10 \%$ du $\mathrm{CA} \mathrm{global}^{98}$.

\section{Conclusion}

À propos de l'alsacien, Dominique Huck s'est posé la question de l'utilité, plus exactement celle «de l'(in)utilité de la langue régionale dans l'entreprise ${ }^{99} »$. S'appuyant sur le témoignage d'un cadre supérieur de 43 ans, chargé de clientèle, il s'est demandé si le dialecte alsacien (que les usagers nomment «l'alsacien ») peut avoir un rôle, notamment économique, dans une entreprise à rayonnement international. Ce témoin, rapporte-t-il, manifeste « une forme de loyauté à sa propre histoire linguistique »: il s'adresse à ses parents en alsacien et essaie de le parler à ses filles. Mais le dialecte n'a pas de statut fonctionnel dans sa vie professionnelle : il lui a facilité l'apprentissage de l'allemand et de l'anglais, mais il n'a pour lui aucune utilité directe dans l'entreprise. D. Huck en conclut que « le dialecte est sans cesse présent, mais invisible [...]. Le dialecte et sa connaissance sont à la fois inutiles dans l'interaction économique en tant que telle, importants comme élément implicite prérequis ${ }^{100} \gg$.

98. Entretien téléphonique du 13 juin 2013.

99. Dominique Huck, «De l'(in)utilité de la langue régionale dans l'entreprise. Déclarations d'un acteur d'une entreprise. Étude de cas », dans Dominique Huck et René Kahn (dir.), Langues régionales, cultures et développement, Paris, L'Harmattan, 2009, p. 233-244.

100. Dominique HucK, « De l'(in)utilité de la langue régionale dans l'entreprise... », ibid. 
Cette étude de cas ne reflète sans doute pas la diversité des usages de langues dans les entreprises d'Alsace ni dans la société prise dans son ensemble. Le même auteur observe par ailleurs une chute tendancielle de la connaissance et de l'usage du dialecte : selon une enquête de 2005, le pourcentage des élèves de moins de 11 ans déclarant pouvoir le parler est de $2,75 \%$, alors que celui de leurs parents s'élève à $35 \%{ }^{101}$. Une autre recherche fait apparaître que «le dialecte n'a plus guère de rôle dans la vie privée des [informateurs], [mais] il est, pour partie au moins, refonctionnalisé dans les entreprises ${ }^{102} »$. Frédéric Mékaoui a cependant pu mettre en évidence que des interactions de proximité ou de connivence peuvent effectivement s'établir entre partenaires économiques (entre vendeur et client, par exemple) dans la langue régionale : l'alsacien est alors fonctionnellement perçu comme pouvant être nécessaire ou utile au sein de l'entreprise, y compris lorsqu'il s'agit d'une entreprise transfrontalière ${ }^{103}$.

Le cas du breton pourrait à la fois s'apparenter à celui du dialecte alsacien et s'en différencier à plus d'un titre. Il s'en différencie par deux caractéristiques au moins. D'une part, son usage fonctionnel constaté lors du sondage de 2007 paraît résiduel dans le monde de l'entreprise au regard de celui qu'avaient observé les lycéens de Guingamp en 1990. D'autre part, le secteur industriel, artisanal ou commercial se signale par le faible nombre des emplois pourvus comme nécessitant la connaissance de la langue. En l'état, les enquêtes de Stumdi et les témoignages collectés corroborent ce premier indicateur. Si, pour ce qui est du critère linguistique, le secteur économique apparaît sous-représenté par rapport aux autres, est-ce parce qu'il ne propose pas de postes ou parce qu'il n'attire pas de candidats? Sous réserve d'inventaire, l'utilisation du breton dans

101. Dominique HuCK, « De l'(in)utilité de la langue régionale dans l'entreprise... », ibid.

102. Arlette Bothorel-Witz et Irini TsAmadou-Jacoberger, « Les processus de minoration et de majoration dans le discours sur les langues et les pratiques dans des entreprises à vocation internationale », dans Dominique Huck et René Kahn (dir.), op. cit., p. 69.

103. Frédéric MéKAOUI, «Langues et emplois dans l'espace du Rhin supérieur. Approche quantitative et subjective », dans Dominique Huck et René Kahn (dir.), op. cit., p. 245-265. 
le cadre du fonctionnement de l'entreprise pourrait être encore plus invisible que celle de l'alsacien. Comme ce dernier, il peut être un élément implicite, mais il n'est pas prérequis.

Il apparaît par contre qu'un certain nombre d'entités, en particulier la grande distribution et la marque "Produit en Bretagne", font un usage ostensible du breton surtout pour l'affichage et éventuellement pour leur publicité. Les grandes surfaces concernées sont moins nombreuses qu'on pourrait le croire ${ }^{104}$, mais les initiatives qu'elles mettent en œuvre en matière de signalisation interne ont indiscutablement pour effet de rendre visible la langue bretonne aux yeux de toute leur clientèle. Cet affichage n'est-il cependant pas que de l'ordre du symbolique ? À quelque exception près, il est toujours bilingue : il ne risque guère de perturber le consommateur qui ignore tout du breton. On peut remarquer en outre que le bilinguisme ne concerne pas les messages qui vont à l'essentiel : les promotions (pour les légumes ou fruits de saison, par exemple), les événements (comme la fête des mères ou des pères) ni les annonces au micro ${ }^{105}$.

Pour sa part, PeB prend soin de diffuser ses prospectus bilingues jusqu'en Loire-Atlantique (tant qu'à faire) et présente comme « une première mondiale » le fait qu'une langue minoritaire y figure en bonne place. La marque est également très fière de la première campagne de publicité " Plijadur penn-da-benn » qu'elle a menée en 2007 dans le métro, à Paris. Ce fut une première en effet, saluée comme telle : « c'est probablement la première fois dans l'Histoire (sic) que la langue bretonne est utilisée comme vecteur de communication de masse, qui plus est, en dehors de la Bretagne ${ }^{106} »$. Elle n'en révélait pas moins une forme d'instrumentalisation du breton, qui n'est plus perçu comme une langue de communication pour ceux qui le parlent et le comprennent, mais comme un simple outil pour intriguer et retenir l'attention d'une population ne la connaissant pas. Il est vrai que le but premier de cette campagne de publicité était de vendre des produits bretons dans la capitale. Depuis, PeB en a orga-

104. Les enseignes de la grande distribution qu'on peut relever en octobre 2014 parmi les signataires de la charte "Ya d'ar brezhoneg », sont au nombre de 8 dans la catégorie "grandes surfaces » : 1 centre Leclerc, 5 Intermarché et 2 Super U.

105. "Qui sera le premier à diffuser des messages audio bilingues en magasin ? ", (Bouëssel, $2012: 33$ ).

106. Y. Rivallain, op. cit., p. 9. 
nisé d'autres, similaires, en Bretagne même et donc aussi en BasseBretagne. Elle a même bretonnisé son logo en « Produet e Breizh» (document 11).

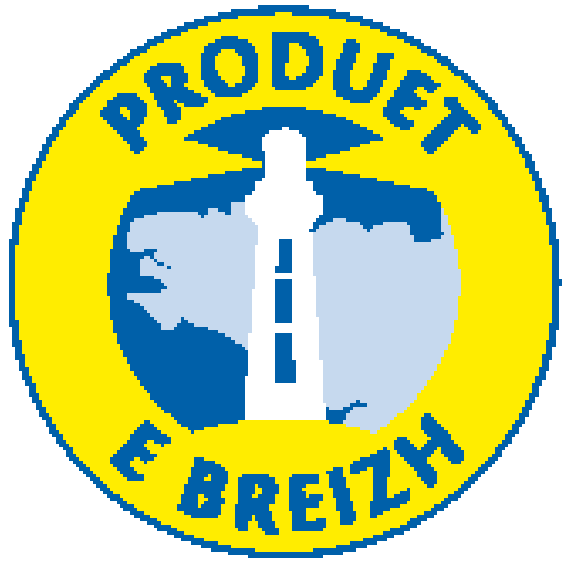

Document 11.

La version en langue bretonne du logo de « Produit en Bretagne ». Des marques comme la bière Coreff l'utilisent.

L'association et ses adhérents reconnaissent que la prise en compte du breton leur a été « très bénéfique en termes d'image ${ }^{107}$ » : il n'y a aucune raison d'en douter. On peut penser qu'elle a été aussi bénéfique pour la langue bretonne, dans le sens où elle a contribué à en valoriser également l'image : c'est une mutation importante par rapport à celle qui prévalait toujours dans le monde de l'économie au début des années 1990 puisqu'elle lui donne de cette manière une visibilité qu'elle n'avait pas auparavant dans l'espace public ${ }^{108}$. Outre qu'elle ne s'étend pas, loin de là, à toute la sphère du commerce et des affaires, cette prise en compte n'en pose pas moins question du point de vue de l'observation des usages de langues.

Contribue-t-elle à l'extension de l'utilisation du breton au quotidien ? Tout ce qui s'entend et se lit en faveur de sa promotion dans le monde de l'économie ne serait-il pas que de l'ordre du discours ${ }^{109}$ ? En réalité, les modalités qui paraissent actuellement prédominantes

107. Y. Rivallain, op. cit., p. 14.

108. En raison de sa fréquentation, l'espace privé du commerce apparaît de facto comme un espace public.

109. En linguistique, l'ensemble des énoncés et des phrases enchaînées formant un message. 
pour ce qui est de son utilisation dans l'entreprise se rapportent principalement à sa mise en image et, pourrait-on dire, à sa mise en scène : elles interviennent essentiellement au niveau de la représentation qu'on se fait de la langue, tout en contribuant à la construire. Sous réserve d'observations complémentaires ${ }^{110}$, elles n'induisent pas ou peu d'interactions fonctionnelles nécessitant de la pratiquer sur le lieu de travail ou pour une transaction commerciale, que ce soit à l'oral ou à l'écrit, si ce n'est sur la base d'un choix volontariste. Sur ce plan précis, le breton pourrait apparaître comme une langue encore plus invisible que l'alsacien : il n'est plus si présent dans la sphère privée, et sauf exception il ne l'est pas fonctionnellement dans l'entreprise. En l'état actuel des choses, il peut représenter un plus, mais l'entreprise n'a généralement pas un besoin imprescriptible de l'utiliser pour son fonctionnement interne ou pour sa relation client.

Comment dès lors l'usage de la langue dans le monde de l'économie pourrait-il représenter une résistance, ou à tout le moins un frein à la substitution, comme peut l'être le développement de l'enseignement bilingue par ailleurs ? Il est vrai que, sous réserve d'inventaire, personne n'a jusqu'à présent présenté aucun projet pour l'entreprise en prenant en compte une telle perspective. $\mathrm{Si}$ l'économique a joué un rôle plus important que le politique dans la mise en place de la substitution au $\mathrm{XX}^{\mathrm{e}}$ siècle, peut-il prétendre à un rôle équivalent au $\mathrm{XXI}^{\mathrm{e}}$ siècle pour l'inversion du changement de langue ? Alain Alcouffe suggère d'analyser le rapport entre les langues régionales et l'économie selon une grille à quatre dimensions, prenant en compte les forces et les faiblesses d'une part, et d'autre part les opportunités et les menaces ${ }^{111}$. Bien des paramètres peuvent en effet interférer à l'avenir dans le cas du breton comme dans celui d'autres langues à statut comparable.

110. Ces informations pourraient être apportées par l'indicateur de l'utilisation de la langue bretonne dans les entreprises qu'a songé à créer PeB. Les bilans de $Y a$ d'ar brezhoneg pourraient également constituer une source documentaire utile.

111. Alain AlCOUfFE, "Économie des langues et politiques linguistiques », dans Georg Kremnitz (dir.), Histoire sociale des langues de France, op. cit., p. 209224. 


\section{ANNEXE}

\section{Témoignage : Le lancement de « Produit en Bretagne»}

Le témoignage qui suit est inédit. Il émane de l'un des deux initiateurs du concept "Certifié Bretagne », dont la dénomination a assez vite évolué en « Produit en Bretagne».

L'histoire de Produit en Bretagne a commencé en novembre 1993, par le lancement du concept « achat citoyen breton». À l'origine : Le Télégramme (qui apportait espace rédactionnel et publicitaire) et le Crédit Mutuel de Bretagne (qui parrainait et finançait). Il s'agissait de susciter «l'achat citoyen breton » en incitant les consommateurs de la région à donner la préférence, à prix et qualité comparables, à des produits élaborés en Bretagne. Dès le 8 novembre 1993, une pleine page du quotidien présentait l'idée fondatrice, ses enjeux pour la Bretagne, l'importance de l'adhésion des Bretons à l'opération... Bien d'autres pages suivront pour, exemples d'entreprises et de marques à l'appui, expliquer aux consommateurs acheteurs que les futurs emplois régionaux se trouvaient pour partie... au fond du caddy.

Au-delà de l'explication, il fallait convaincre quelques entreprises de se prêter à la démonstration, de diverses manières, pour amorcer le processus. Avec la Scarmor/Leclerc, quelques entreprises agroalimentaires ont immédiatement adhéré à l'idée : Even, S.I.L.L., Unicopa, Rolland/ Flipi, CECAB, Petit Navire, Doux... Le « signe de ralliement » était un autocollant conforme à celui qui illustre la page du site internet actuel de « Produit en Bretagne », mais la mention était « Certifié Bretagne » et non pas encore « Produit en Bretagne».

Rapidement, il est apparu que l'opération ne pouvait prendre toute son envergure et se pérenniser qu'à certaines conditions :

- que les initiateurs abandonnent toute prétention à droit d'antériorité ou d'exclusivité. Ainsi, Le Télégramme devait, pour que l'opération puisse s'étendre au-delà de sa zone de diffusion (Finistère et arrondissements limitrophes des Côtes d'Armor et du Morbihan), accepter que Ouest-France la relaie à son tour mais, cette fois, sur les cinq départements de la Bretagne historique. De son côté, le CMB devait admettre que le Crédit Agricole, puis les autres établissements bancaires bretons entrent aussi dans l'opération. Quant au groupement Leclerc/Scarmor, il acceptait que d'autres distributeurs se joignent au projet. Ce qui fut rapidement acté, entre J.-Y. Chalm (Le Télégramme), J. Bannier (CMB), J.-C. Simon (Even) et C. Pujol (Scarmor) ; 
- que les industriels et les distributeurs prennent les rênes de l'opération et que soit créée une association, dont J.-C. Simon fut le premier président, et qu'elle soit professionnalisée : une déléguée générale salariée fut rapidement recrutée, grâce à l'aide financière des collectivités régionales négociée notamment par G. Falc'hun. Parallèlement, les premiers collèges étaient créés : Industriels et Distributeurs donc, mais aussi Services (banques et... transporteurs, au début) tandis que les deux quotidiens régionaux étaient cooptés comme membres associés ;

- dernière condition : que soit abandonné le nom «Certifié Bretagne » qui pouvait, au regard de la réglementation, poser problème car évoquant une certification ou un label. D'où l'apparition, deux après, de l'appellation « Produit en Bretagne » et du logo actuel avec phare et faisceau.

Tout cela s'est déroulé très naturellement et rapidement, dans une forme d'enthousiasme et de partage d'ambitions pour la Bretagne, pour les entreprises et pour l'emploi régional, objectif originel et évidemment prioritaire. Bien sûr, la réussite de l'opération, illustrée par la hausse parfois spectaculaire du chiffre d'affaires généré par les produits stickés «Certifié en Bretagne » puis " Produit en Bretagne ", a puissamment contribué à entretenir cet enthousiasme et le dynamisme de l'association.

Pour retrouver toutes les traces de cette histoire encore récente, il suffit de se reporter aux pages du Télégramme d'il y a un peu plus de 20 ans ${ }^{1}$. Explication du projet dans les colonnes du quotidien et partenaire initial, incitation à acheter breton, logo " Certifié Bretagne », premières entreprises contactées, premiers engagements sur la création d'emploi... Tout y est.

Jean Bannier ${ }^{2}$

20 août 2014

1. Au sommaire des pages que Le Télégramme consacre au lancement de «Certifié Bretagne » le 8 novembre 1993, on peut lire les articles suivants :

- Certifié Bretagne. Valoriser nos talents.

- Le Centre Bretagne à la reconquête de son marché intérieur.

- Le paysage breton reste imprégné du poids de l'agro-alimentaire.

- OTOR Carhaix : ses cartons emballent.

- La consommation de l'an 2000 : le triomphe de l'individu.

- Le bon exemple vient du Québec.

- Géminox : chaud devant.

On remarquera que le slogan qui figure sous le logo « Certifié Bretagne » précise que « Produire et acheter en Bretagne, c'est créer des emplois ».

2. Jean Bannier était en 1990 le directeur de la Communication du CMB (Crédit Mutuel de Bretagne). Il deviendra ensuite Directeur Général Adjoint du groupe Crédit Mutuel Arkéa. 